GEOLOGICAL SURVEY CIRCULAR 810

\title{
The Magnetic Charts of the
}

United States for

Epoch 1975

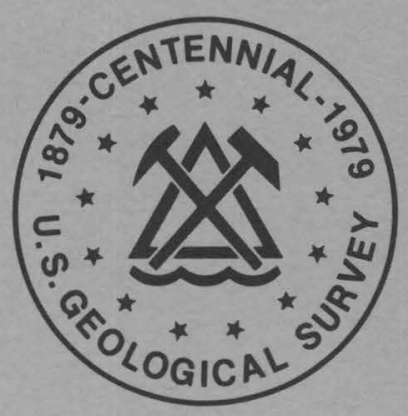





\section{The Magnetic Charts of the}

United States for

Epoch 1975

By E. B. Fabiano, W. J. Jones, and N. W. Peddie

GEOLOGICAL SURVEY CIRCULAR 810 
United States Department of the Interior

CECIL D. ANDRUS, Secretary

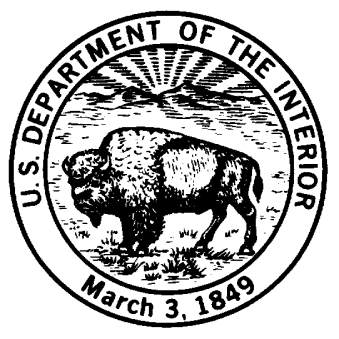

\section{Geological Survey}

H. William Menard, Director 
Abstract.................................................. 1

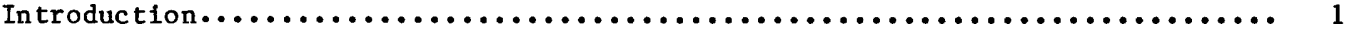

Procedures $. \ldots \ldots \ldots \ldots \ldots \ldots \ldots \ldots \ldots \ldots \ldots \ldots \ldots \ldots \ldots \ldots \ldots \ldots \ldots \ldots \ldots \ldots \ldots \ldots \ldots \ldots \ldots \ldots \ldots \ldots \ldots . \ldots 1$

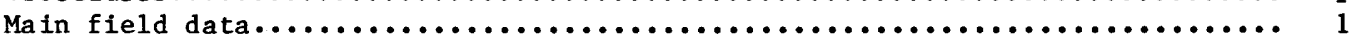

Secular variation data..........................................

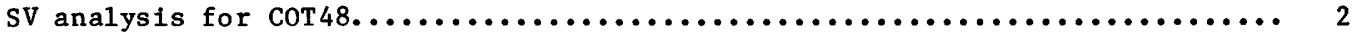

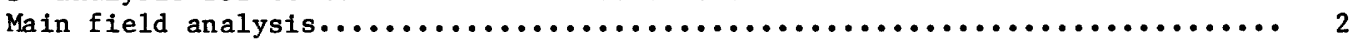

D models for CoT 48 and Alaska...................................

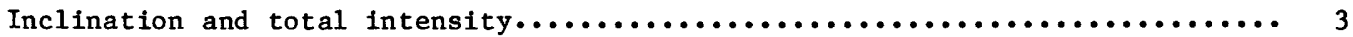

Model coefficients and final charts..............................

Evaluation and discussion.....................................

Conclusion................................................ 4

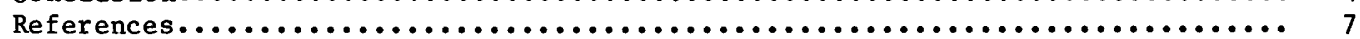

\section{APPENDIXES}

A: The number of $\mathrm{H}$ and $\mathrm{Z}$ survey measurements by one degree tessera within

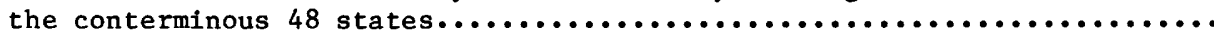

B: Residuals--U.S. repeat stations and observatories, including Alaskan repeat stations........................................... 11

C: Coefficients for chart models................................. 14

\section{ILLUSTRATIONS}

Figure 1: Total intensity in the United State for 1975................

2: Comparison of FDOT 1965 and 1975 United States magnetic charts.... 6

TABLES

Table 1. Statistics of fit of SV models to initial linear rates of change at

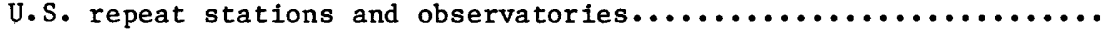

2. Main field rms deviations for the one-degree tessera means.........

3. Summary of rms deviations of main field models with subsets of survey data using models of $n=7 . \ldots \ldots \ldots \ldots \ldots \ldots \ldots \ldots \ldots \ldots \ldots \ldots \ldots$

4. Summary of rms deviations of the fit of models with repeat stations

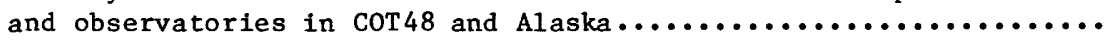

5. Summary of rms deviations of $D$ models to tessera means of declination......................................... 


\title{
THE MAGNETIC CHARTS OF THE UNITED STATES
}

\section{FOR EPOCH 1975}

\author{
By E. B. Fabiano, W. J. Jones, and N. W. Peddie
}

\begin{abstract}
Approximately 34,000 measurements taken
\end{abstract} from 1900 to 1974 were analyzed by least-squares methods to produce a series of five magnetic charts of the United States for 1975. A feature of the analysis, differing from techniques used for previous editions of the national chart, is that analytic models define the regional magnetic field and are used to contour the magnetic charts.

An overall rms (root mean square) fit of less than $235 \mathrm{nT}$ was obtained for the horizontal and vertical intensities; for the chart of magnetic declination, the rms deviation was on the order of $0.5^{\circ}$. The models of annual change, derived from data at repeat stations and observatories operated by the U.S. Geological Survey, yielded an rms deviation of approximately $6 \mathrm{nT} / \mathrm{yr}$ in the force components and $0.7 \mathrm{~min} / \mathrm{yr}$ in magnetic declination.

\section{Introduction}

This report describes the method used to produce the magnetic charts of the United States for 1975, which were published by the U.S. Geological Survey in cooperation with the National Oceanic and Atmospheric Administration (Fabiano and Jones, 1976; Fabiano and others, 1976; Peddie and others, 1976; Jones and others, 1976; Fabiano, 1975). The charts are a continuation of a series that began with the isogonic chart for 1850 and, with a few exceptions, have been published at 5- and 10year intervals since then. Deel and Howe (1948) and Svendsen (1962) provided historical summaries related to the magnetic surveys and charts of the United States. In 1975, five charts were published: declination (D), inclination (I), horizontal intensity (H), vertical intensity ( $\mathrm{Z}$ ), and total intensity (F). The general objective for these charts was to provide the users of geomagnetic data, such as surveyors and navigators, with references depicting the smoothed distribution of the five components of the Earth's magnetic field in the region of the United States. A secondary objective, related to techniques, was to determine if the regional field could be adequately modeled using simple analyt ic expressions which subsequently could be used as a convenient means for contouring the isolines and also for synthesizing chart values for varied user needs.

\section{Procedures}

The charts for magnetic declination, $D$, are discussed in a later part of this report. The following brief outline describes the procedures used to obtain the $I, H, Z$, and $F$ charts:

1. Determine models of current secular change for data reduction.

2. Reduce magnetic survey measurement $s$ of $\mathrm{H}$ and $\mathrm{Z}$ ( $t$ aken from 1900 through 1974) to epoch 1975.

3. Delete from the reduced file those measurements which appear to be either in error or highly anomalous.

4. Obtain one-degree tessera means of $H$ and $Z$.

5. Perform separate polynomial analysis of $\mathrm{H}$ and $\mathrm{Z}$ for the conterminous 48 States region (COT48) and for Alaska. For Hawail, copy the results from AWC75, the model obt ained to create the American World Charts for 1975 (Peddie and Fabiano, 1976).

6. Evaluate and adopt final models; prepare magnetic charts.

\section{Main Field Data}

The initial file of magnetic survey data consisted of measurements at 26,097 locations 
within the COT48 region and 8,036 locations in Alaska. A record for each location was considered acceptable only if both $\mathrm{H}$ and $\mathrm{Z}$ were measured at the station. Some 70 percent of the data were measured prior to 1963 , and more than one-half of the entire file were obtained from the airborne surveys conducted by the U.S. Naval Oceanographic Office and the Department of Energy, Mines and Resources of Canada. Airborne survey data now constitute more than 85 percent of the survey file measured since 1953.

The file of measurements obtained from 1900 to 1962 had been previously reduced for the 1965 series of magnetic charts. Consequently, the reduction procedure for this subset of data involved corrections for the last few years of the previous update $(1962.5-1965.0)$ and the application of the more recent secular variation until the epoch date of 1975.0. Aeromagnetic data were corrected to sea level using an approximation suggested by Chapman (1951). For small heights (h) measured in kilometers, any component will be decreased by a fraction $h / 2123$ of itself at height h.

To identify and remove what appear to be erroneous or anomalous values, residuals were computed for each measurement using the world magnetic charts model for epoch 1970 (Hurwitz and others, 1974) as the reference field and 1967.5 as the reference date. Data with residuals exceeding $800 \mathrm{nT}$ ( $1,000 \mathrm{nT}$ for Alaska) were rejected and, as a result of this screening process, 12 percent of the original file was omitted from the analysis.

Appendix A shows the distribution of $H$ and $Z$ observations used in obtaining the tessera means for the COT48 region. The gaps represent the areas where no $H$ and $Z$ measurements were available. For Alaska, since one-third of the area lacked vector data, values were synthesized from AWC75 and used to supplement this file for each $2^{\circ}$ by $2^{\circ}$ gap.

Because relatively few $D$ measurements had been acquired since the last series of $D$ charts, published in 1970, the decision was made to synthesize one value for each one-degree quad from these charts, apply SV (secular variation) corrections from 1967.5-1975.0 and then perform the least-squares analysis.

\section{Secular Variation Data}

The file used for the SV analysis consisted of three categories of data:

1. The annual mean values of $D, H$, and $\mathrm{Z}$ from Canadian and U.S. magnetic observatories.

2. Vector field measurements at some 100 repeat stations in COT 48 , which are occupied every 5-7 years.

3. Supplemental values of annual change, synthesized from AWC 75. These consisted of values on a $3^{\circ} \times 3^{\circ} \mathrm{grid}$ in the ocean areas and were included to minimize edge effects at the chart borders.

At the time this chart was being compiled, no repeat station data since 1965 were available on time to perform a regional SV analysis for Alaska. Therefore, for Alaska, grid values of annual change from AWC 75 were used to derive the SV polynomial coefficients. (Appendix B lists the Alaskan repeat stations which, nevertheless, were useful in the subsequent evaluation.)

\section{SV Analysis for COT 48}

For the intervals 1962.5-1967.5 and 1967.5-1975.0, the linear rates of change for $D$, $H$, and $Z$ were determined by straight lines fitted to the OAM (observatory annual means) and the repeat-station measurements. Data were reviewed for errors, and on the basis of a preliminary analysis, the observatory $r$ ates were given seven times greater weight than the repeat-station rates.

The function adopted for the least-squares procedure is of the form

$$
\left.K=\sum_{i=0}^{n} \stackrel{\sum}{=}=0_{i j}^{1} a_{c}^{i-j}\right)\left(\lambda_{c}^{j}\right) \text {, }
$$

where $K$ is the linear rate of change; $n$, the degree of the polynomial; $a_{1 j}$, the coefficlents to be determined; and $\theta, \lambda$, the normalized geographic coordinates. For COT48, coordinates were normalized so that $\theta_{c}=\theta-52^{\circ}$ and $\lambda_{c}$ $=\lambda-268^{\circ}$. Here, $\theta=$ colatitude and $\lambda=$ east longitude.

Analyses were performed for degrees 4 and 6 (15 and 28 coefficients respectively). On the basis of the rms deviations, shown in table 1 , the latter models were adopted for final use.

Table 1.-Statistics of fit of $S V$ models to initial linear rates of change at U.S. repeat stations and observatories

[SV1, model for 1962.5-1967.5; SV2, model for 1967.5-1975.0. Column entries show the rms deviations. Leaders $(---)$ indicate no data ]

\begin{tabular}{ccccc}
\hline Degree & Mode1 & $\begin{array}{c}\mathrm{D} \\
(\mathrm{min} / \mathrm{yr})\end{array}$ & $\begin{array}{c}\mathrm{H} \\
(\mathrm{nT} / \mathrm{yr})\end{array}$ & $\begin{array}{c}\mathrm{Z} \\
(\mathrm{nT} / \mathrm{yr})\end{array}$ \\
\hline \multirow{4}{*}{6} & SV 1 & -- & 5.2 & 6.4 \\
& SV 2 & 0.8 & 5.5 & 7.1 \\
& SV 1 & -- & 4.3 & 5.8 \\
& SV 2 & .7 & 4.7 & 6.4 \\
\hline
\end{tabular}

Main Field Analysis

Values of $\mathrm{H}$ and $\mathrm{Z}$ were averaged to obtain means for each one-degree tessera, and the data were subsequently analyzed using polynomial functions of the form described in the section entitled "SV Analys is for COT48." Tests were run for $n=4,7$, and 9, with the objective of determining the optimum fit with the fewest number of coefficients. Finally, the models for $\mathrm{n}=7$ were adopted.

For Alaska, the analysis presented two difficulties:

1. The data-selection region for Alaska, consisting of 2,556 one-degree 
tesserae, contained acceptable $\mathrm{H}$ and $Z$ data for 1,675 tesserae, only 66 percent of the region.

2. Because of the chart projections used, the COT48 area in the northwest overlaps the region on the Alaska side of the same chart. Since the COT48 and Alaskan models were to be derived separately, it was necessary to insure that the models were consistent in the region of overlap.

Consequently, fill-in grid values were synthesized from the AWC 75 model for the sparsely surveyed region and from the COT48 models of $\mathrm{H}$ and $\mathrm{Z}$ in the overlap area. This amalgamated data set for Alaska, consisting of both sets of synthesized values and the onedegree tessera means, was then analyzed to produce degree-7 polynomial models for $H$ and $Z$, using colatitude $32^{\circ}$ and east longitude $205^{\circ}$ to normalize the coordinates.

\section{Models for COT48 and Alaska}

The $\operatorname{coT} 48$ region was partitioned into five areas, each consisting of 12-degree longitudinal bands. Separate analyses, degrees 4 and 7 , were performed for each band, allowing for 2-degree longitudinal overlaps at the partition boundaries to minimize edge-effect distortions.

For Alaska, a single model $n=7$ for the entire region was obtained from values synthesized and updated from the 1970 Alaska magnetic chart (U.S. Department of Commerce, 1970 ) and additional fill-in values from the COT48 and AWC75 models.

\section{Inclination and Total Intensity}

The charts of $I$ and $F$ were derived from the $\mathrm{H}$ and $\mathrm{Z}$ models using the formulas

$$
I=\arctan \mathrm{Z} / \mathrm{H} \text { and } \mathrm{F}=\left(\mathrm{H}^{2}+\mathrm{Z}^{2}\right)^{1 / 2}
$$

The annual change rates, $\dot{I}$ and $\dot{F}$, were computed from the first derivatives, where

$$
\begin{aligned}
& \dot{\mathrm{I}}=(\mathrm{H} \dot{\mathrm{Z}}-\mathrm{Z} \dot{\mathrm{H}}) /\left(\mathrm{H}^{2}+\mathrm{Z}^{2}\right) \text { and } \\
& \dot{\mathrm{F}}=(\mathrm{H} \dot{\mathrm{H}}+\mathrm{Z} \dot{\mathrm{Z}}) /\left(\mathrm{H}^{2}+\mathrm{Z}^{2}\right)^{1 / 2} \text {. }
\end{aligned}
$$

\section{Mode1 Coefficients and Final Charts}

The coefficients for the components $D, H$, and $Z$, shown in Appendix $C$, was subsequently used to contour the five magnetic components. Where required, near the chart borders, isolines were manually adjusted to merge with the corresponding isolines of the American World Magnetic Charts for 1975. Consequently, we recommend that for synthesizing values from the analyt ic models, one should use the American World Charts for ocean areas $200 \mathrm{~km}$ beyond the U.S. coastlines.
Evaluation and Discussion

Tables 2, 3, and 4 summarize the major statistics pertinent to the evaluation of the models. In table 2, the lower rms deviations shown for degree 7 are what one may anticipate from an analysis of averaged values.

In $t a b l e 3$, the adopted models for degree 7 are compared to the survey data file. The slightly larger rms deviations for COT48 column A (compared to column B) probably result from the fact that $A$ contains the older data. However, the differences are not large and are within the probable error limits for measured data. Both tables suggest that the COT48 and Alaska charts have comparable accuracies.

The most recent data available for determining the quality of the fit to the models are the vector measurements at the magnetic observatories and repeat stations for the interval 1971-74. The residuals for these stations, shown in Appendix B, are applicable for the epoch date of the measurement indicated in the last column; the summarized statistics are shown in table 4.

Measurements from repeat stations and observatories represent the most reliable survey data available. By comparison with other measurements, they are only marginally affected by temporal and transient variations and generally the stations are located in nonanomalous regions. As a result, these corresponding rms fits are quite good and serve to substantiate the rms fits shown in tables 2 and 3 .

\begin{tabular}{|c|c|c|c|c|}
\hline \multirow[b]{2}{*}{ Degree ------- } & \multicolumn{3}{|c|}{ COT48 } & \multirow{2}{*}{$\frac{\text { Alaska }}{7}$} \\
\hline & 4 & 7 & 9 & \\
\hline Component: & & & & \\
\hline $\mathrm{H}$ & 156 & 118 & 116 & 115 \\
\hline $\mathrm{Z}$ & 152 & 130 & 128 & 148 \\
\hline
\end{tabular}

Table 2.-Main field rms deviations, in nanoteslas, for the one-degree tessera means (units of $n T$ )

Table 3.-Summary of $r$ s deviations of main field models with subsets of survey data using models of $n=7$ (units of $n T$ )

\begin{tabular}{ccccc}
\hline & \multicolumn{2}{c}{ A } & B & C \\
\cline { 2 - 5 } Component & \multicolumn{2}{c}{ COT48 } & Alaska \\
\cline { 2 - 5 } & (Every 5th value, & (A11 values, & & (All values, \\
& $1900-74)$ & $1900-74)$ \\
\hline H & 188 & 188 & 184 \\
Z & 226 & 197 & 234 \\
\hline Number of values & 4,409 & 15,420 & 7,881 \\
\hline
\end{tabular}

Table 4.-Summary of rms deviations of the fit of models with data from repeat stations and observatories in COT48 and Alaska [COT48, 113 stations; Alaska, 26 stations]

\begin{tabular}{cll}
\hline Component & C0T48 & Alaska \\
\hline D & $0.37^{\circ}$ & $0.52^{\circ}$ \\
H & $145 \mathrm{nT}$ & $106 \mathrm{nT}$ \\
$\mathrm{I}$ & $0.18^{\circ}$ & $0.13^{\circ}$ \\
$\mathrm{Z}$ & $225 \mathrm{nT}$ & $184 \mathrm{nT}$ \\
$\mathrm{F}$ & $205 \mathrm{nT}$ & $174 \mathrm{nT}$ \\
\hline
\end{tabular}


Table 5 refers to the partitioning scheme and related statistics for the $D$ models of COT48. Because the values analyzed are scaled from a smooth chart, the overall fit, as one might anticipate, is remarkably close, to within 6 minutes of arc for most partitions, providing some evidence for the adequacy of the modeling process for regions as large as 2 million square kilometers--the approximate size of each partitioned area. The larger rms values in bands 2 and 3 probably indicate the numerous declination anomalies in the Great Lakes region.

Table 5.-Summary of rms deviations of D models to tessera means of declination

[ $\gamma$ column shows the east longitude normalizing factor]

\begin{tabular}{|c|c|c|c|c|}
\hline \multirow{3}{*}{ Band } & \multirow{3}{*}{$\begin{array}{l}\text { Partition } \\
{ }{ }_{W} \text {. long }\end{array}$} & \multicolumn{2}{|c|}{ rms (in degrees) } & \multirow{3}{*}{$\begin{array}{c}\gamma \\
\text { (1n degrees) }\end{array}$} \\
\hline & & \multicolumn{2}{|c|}{ Degree } & \\
\hline & & 4 & 7 & \\
\hline 1 & $66-77$ & 0.11 & 0.01 & 289 \\
\hline 2 & $78-89$ & .18 & .14 & 277 \\
\hline 3 & $90-101$ & .14 & .10 & 265 \\
\hline 4 & $102-113$ & .08 & .06 & 253 \\
\hline 5 & $114-125$ & .06 & .05 & 241 \\
\hline
\end{tabular}

The D models were also evaluated by comparing them to two subsets of survey data. The first set consisted of 1,450 station values, updated to 1975.0 and randomly distributed within COT48. From these measurements, an overall rms of $0.50^{\circ}$ was determined. The second subset, comprising survey data excluded from the file of analysis data, consisted of 287 airborne measurements from the Project Magnet surveys made from 1967 to 1970. With this set, the computed rms was $0.52^{\circ}$.

Among the reasons for the lack of $\mathrm{fit}$ that one may observe from the differences which exist between the data and models, the following should be noted:

1. Errors in the data. This problem was discussed earlier. Wherever possible, it is important to determine whether the errors are random or systematic. Hannaford and Haines (1969), for example, reported probable errors in aeromagnetic data of $0.4^{\circ}$ in $D$, $40 \mathrm{nT}$ in $\mathrm{H}$, and $70 \mathrm{nT}$ in $\mathrm{Z}$. In the older land survey data, Deel and Howe (1948) reported possible errors as large as $150 \mathrm{nT}$ in the computed value of $Z$ due to instrumental uncertainties.

2. Secular variation. The secularvariation estimates for the earlier years, especially prior to the $1930^{\prime} \mathrm{s}$, are less reliable, due primarily to insufficient data as well as to the quality of the instrument ation. In addition, the use of linear SV estimates, though convenient and in some instances practical, introduces errors in data reduction because the $\mathrm{SV}$ is not necessarily linear. This is not a serious problem for datareduction intervals of a few years. However, for a 5- or 10- year interval, significant errors could accumulate.

3. Local and regional anomalies. It is difficult at this time to assess the impact resulting from the local and

regional magnetic anomalies on the statistics relating to the fit of the data to the model. If, for example, one had available up-todate vector measurements spaced a few kilometers apart nationwide, relatively accurate assessments of the "normal" and anomalous fields could be made. To date, however, the available vector data are too poorly distributed, both in space and in time.

A small-scale chart of total intensity and its annual change for 1975 in the United States is shown in figure 1. This chart was adapted from the 1975 U.S. total intensity chart (Fabiano and others, 1976). Figure 2 illustrates how SV estimates of total intensity (FDOT) can change over a 10-year period. Note that at latitude $45^{\circ}$ and longitude $90^{\circ}$ the estimated rates of $\mathrm{SV}$ differ by more than $60 \mathrm{nT} / \mathrm{yr}$.

\section{Conclusion}

The various statistics of fit presented indicate that the polynomial technique is generally adequate for producing models of the smoothed field suitable for national reference charts. The major deterrent for obtaining more reliable models appears to lie not so much in technique as it does in the accuracy of the available data base.

Dependence on these data may soon be minimized and perhaps eliminated as a result of two magnetic surveys, one completed and the other planned. During 1977-78, the U.S. Naval Oceanographic office completed a vector survey of the United States with north-south tracklines flown at approximately 100-km spacing; several east-west lines were also flown. In 1979 the National Aeronautics and Space Administration in cooperation with the U.S. Geological Survey will launch MAGSAT, a low-level satellite which will obtain worldwide vector data. The acquisition of measurements from both sources will substantially improve the data base used for future national charts. 


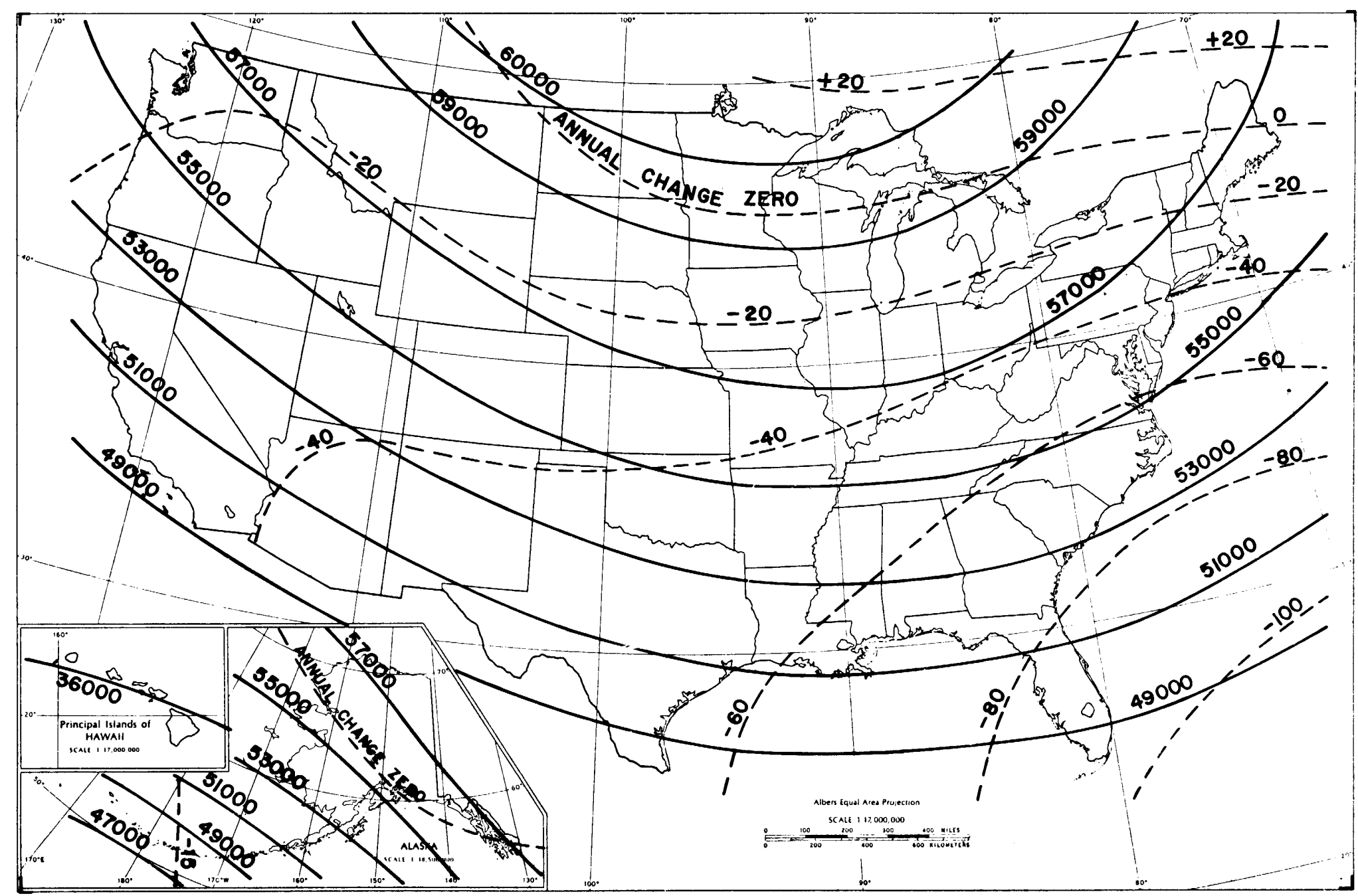

Figure 1. Total Intensity in the United States for 1975. (Solid lines are field contours in units of nanotes1a (nT); dashed lines are annual change contours in units of $\mathrm{nT} / \mathrm{yr}$. ) 


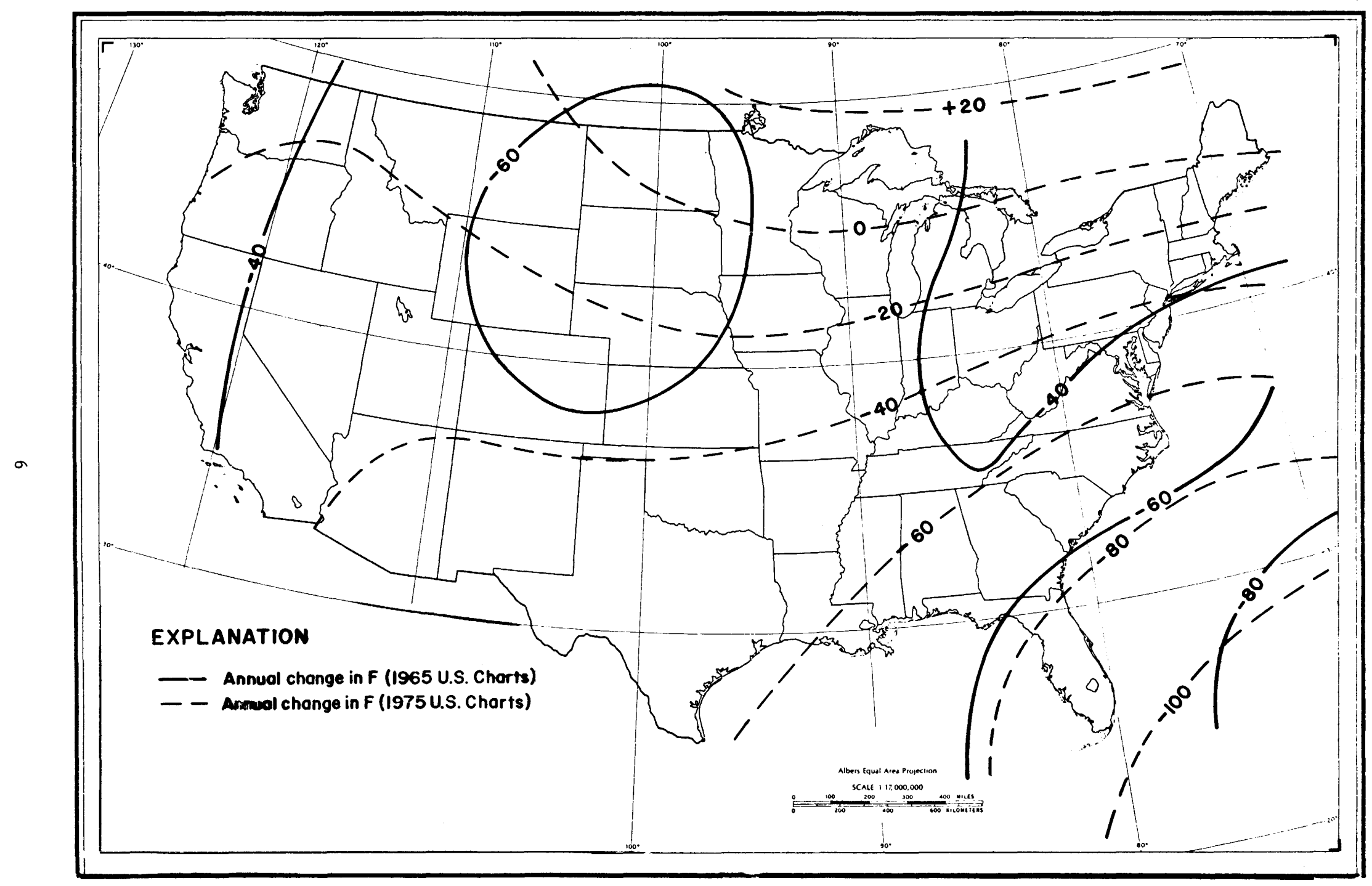

Figure 2. Comparison of FDOT 1965 and 1975 United States magnetic charts (in units of nT/yr.) 


\section{References}

Chapman, Sydney, 1951, The earth's magnetism, (2d ed.): New York, John Wiley, p. 27.

Deel, S. A., and Howe, H. H., 1948, United States magnetic tables and magnetic charts for 1945, U.S. Coast and Geodetic Survey Serial 667: U.S. Government Printing Office, Washington, D.C., P. 10-12.

Fabiano, E. B., 1975, Magnetic declination in the United States, epoch 1975.0: U.S. Geological Survey Miscellaneous Geologic Investigations Map I-911.

Fabiano, E. B., and Peddie, N. W., 1975, U.S. world chart model coefficients for 1975: American Geophysical Union Transactions, v. 56, no. 9, p. 587-588.

Fabiano, E. B., and Jones, W. J., 1976, Magnetic horizontal intensity in the United States, epoch 1975.0: U.S. Geological Survey Miscellaneous Geologic Investigations Map I913.

Fabiano, E. B., Peddie, N. W., and Jones, W. J., 1976, Magnetic total intensity in the United States, epoch 1975.0: U.S. Geological Survey Miscellaneous Geologic Investigations Map I915.

Hannaford, W., and Haines, G. V., 1969, A threecomponent aeromagnetic survey of the Nordic countries and the Greenland sea: Dominion
Observatory Publications, ottawa, v. 37, no. 5, p. 113-164.

Huritz, Louis, Fabiano, E. B., and Peddie, N. W., 1974, A model of the geomagnetic field for 1970: Journal of Geophysical Research, v. 79, no. 11, p. 1716-1717.

Jones, W. J., and Fabiano, E. B., 1976, Magnetic vertical intensity in the United States, epoch 1975.0: U.S. Geological Survey Miscellaneous Geologic Investigations Map I914.

Peddie, N. W., and Fabiano, E. B., 1976, A model of the geomagnetic field for 1975: Journal of Geophysical Research, v. 81, no. 14, p. 2539-2542.

Peddie, N. W., Jones, W. J., and Fabiano, E. B., 1976, Magnetic inclination in the United States, epoch 1975.0: U.S. Geological Survey Miscellaneous Geologic Investigations Map I-912.

Svendsen, K. L., 1962, United States Magnetic Tables for 1960, U.S. Coast and Geodetic Survey Publication 40-2: U.S. Government Printing Office, Washington, D.C., 91 p.

U.S. Department of Commerce, 1970, Isogonic chart of the United States, 1970: U.S. Dept. of Commerce, Enviromental Science Services Administration, chart no. 3077. 
Appendix A.--The number of $H$ and $Z$ survey measurements by one-degree tessera within the conterminous 48 States

[Latitude and longitude, in degrees, are shown in the extreme left-hand column and top row, respectively]

55

54

53

52

51

50

49

48

47

46

45

44

43

42

41

40

39

38

37

36

35

34

33

32

31

30

29

28

27

26

25

24

23

22

21

20

$\begin{array}{llllllllllllllllllllllllll}-130 & -129 & -128 & -127 & -126 & -125 & -124 & -123 & -122 & -121 & -120 & -119 & -118 & -117 & -116 & -115 & -114 & -113 & -112 & -111 & -110 & -109 & -108 & -107 & -106\end{array}$ $\begin{array}{rrrrrrrrrrrrrrrrrrrrrrrrrrrrrrrrrrr}9 & 7 & 6 & 6 & 6 & 9 & 2 & 4 & 10 & 15 & 17 & 21 & 12 & 11 & 8 & 9 & 9 & 15 & 7 & 5 & 4 & 7 & 8 & 3 \\ 9 & 12 & 14 & 12 & 14 & 13 & 6 & 7 & 11 & 8 & 10 & 19 & 17 & 17 & 17 & 16 & 87 & 19 & 10 & 5 & 6 & 3 & 6 & 4 \\ 5 & 10 & 3 & 11 & 4 & 8 & 16 & 10 & 13 & 12 & 7 & 18 & 20 & 18 & 11 & 25 & 26 & 16 & 10 & 20 & 37 & 10 & 7 & 11 \\ 6 & 14 & 14 & 6 & 8 & 7 & 15 & 9 & 7 & 8 & 8 & 21 & 6 & 7 & 9 & 22 & 35 & 12 & 14 & 12 & 21 & 35 & 26 & 32 \\ 13 & 11 & 12 & 7 & 9 & 5 & 10 & 13 & 12 & 10 & 9 & 15 & 11 & 8 & 42 & 36 & 21 & 12 & 9 & 6 & 10 & 11 & 18 & 11\end{array}$ $\begin{array}{lllllllllllllllllllllllllll}11 & 15 & 17 & 20 & 9 & 6 & 8 & 17 & 20 & 23 & 19 & 12 & 13 & 13 & 9 & 16 & 34 & 15 & 21 & 22 & 12 & 19 & 20 & 28\end{array}$ $\begin{array}{llllllllllllllllllllllllll}12 & 12 & 7 & 8 & 13 & 39 & 27 & 14 & 22 & 9 & 21 & 12 & 17 & 13 & 22 & 11 & 28 & 17 & 11 & 14 & 7 & 4 & 5 & 8\end{array}$ $\begin{array}{lllllllllllllllllllllllllll}10 & 13 & 13 & 12 & 10 & 16 & 32 & 17 & 5 & 3 & 8 & 12 & 7 & 12 & 11 & 6 & 18 & 7 & 13 & 9 & 15 & 9 & 11 & 10\end{array}$ $\begin{array}{lllllllllllllllllllllllllll}4 & 4 & 4 & 6 & 4 & 5 & 0 & 63 & 4 & 8 & 4 & 11 & 11 & 6 & 4 & 3 & 1 & 1 & 0 & 2 & 2 & 1 & 0 & 2\end{array}$ $\begin{array}{lllllllllllllllllllllllllll}2 & 0 & 0 & 0 & 5 & 8 & 6 & 3 & 0 & 8 & 4 & 4 & 5 & 0 & 1 & 2 & 11 & 18 & 2 & 2 & 2 & 1 & 1 & 0\end{array}$ $\begin{array}{lllllllllllllllllllllllllll}1 & 3 & 3 & 6 & 9 & 8 & 12 & 18 & 13 & 6 & 2 & 15 & 3 & 3 & 0 & 0 & 2 & 4 & 1 & 4 & 6 & 9 & 3 & 1\end{array}$ $\begin{array}{llllllllllllllllllllllllllllll}0 & 2 & 5 & 6 & 7 & 9 & 20 & 3 & 3 & 3 & 1 & 5 & 6 & 18 & 0 & 0 & 0 & 2 & 0 & 0 & 2 & 3 & 1 & 11\end{array}$ $\begin{array}{llllllllllllllllllllllllll}6 & 4 & 0 & 4 & 3 & 10 & 8 & 2 & 2 & 0 & 4 & 2 & 1 & 8 & 4 & 2 & 1 & 5 & 8 & 3 & 3 & 4 & 1 & 3\end{array}$ $\begin{array}{llllllllllllllllllllllllllllll}3 & 0 & 0 & 2 & 3 & 11 & 4 & 2 & 3 & 2 & 1 & 0 & 0 & 0 & 0 & 15 & 6 & 11 & 4 & 1 & 0 & 3 & 0 & 5\end{array}$ $\begin{array}{llllllllllllllllllllllllllll}0 & 4 & 2 & 2 & 0 & 14 & 3 & 16 & 2 & 2 & 0 & 1 & 2 & 0 & 0 & 1 & 0 & 1 & 18 & 2 & 12 & 4 & 12 & 11\end{array}$ $\begin{array}{lllllllllllllllllllllllllll}5 & 2 & 1 & 6 & 2 & 14 & 2 & 7 & 2 & 4 & 4 & 3 & 15 & 2 & 10 & 10 & 12 & 16 & 27 & 16 & 22 & 17 & 13 & 20\end{array}$ $\begin{array}{llllllllllllllllllllllllll}4 & 5 & 3 & 1 & 2 & 11 & 16 & 3 & 6 & 5 & 19 & 11 & 14 & 12 & 19 & 14 & 11 & 4 & 12 & 14 & 2 & 12 & 9 & 10\end{array}$ $\begin{array}{lllllllllllllllllllllllll}8 & 8 & 8 & 4 & 5 & 2 & 14 & 8 & 12 & 13 & 13 & 13 & 14 & 11 & 16 & 13 & 12 & 14 & 13 & 16 & 9 & 8 & 12 & 10\end{array}$ $\begin{array}{lllllllllllllllllllllllllll}5 & 5 & 5 & 9 & 16 & 25 & 43 & 63 & 22 & 22 & 24 & 31 & 22 & 12 & 12 & 16 & 22 & 20 & 14 & 17 & 21 & 20 & 25 & 21\end{array}$ $\begin{array}{lllllllllllllllllllllllllll}4 & 3 & 13 & 23 & 32 & 32 & 10 & 11 & 5 & 12 & 10 & 4 & 3 & 10 & 20 & 4 & 0 & 2 & 0 & 0 & 0 & 1 & 1 & 2\end{array}$ $\begin{array}{lllllllllllllllllllllllllll}11 & 20 & 24 & 14 & 9 & 15 & 2 & 4 & 5 & 17 & 7 & 3 & 2 & 2 & 1 & 1 & 5 & 13 & 4 & 4 & 3 & 4 & 3 & 11\end{array}$ $\begin{array}{lllllllllllllllllllllllllll}13 & 10 & 7 & 3 & 2 & 6 & 4 & 2 & 18 & 15 & 16 & 17 & 24 & 5 & 4 & 2 & 4 & 9 & 6 & 10 & 5 & 6 & 4 & 9\end{array}$ $\begin{array}{llllllllllllllllllllllll}4 & 0 & 1 & 2 & 4 & 5 & 13 & 18 & 11 & 6 & 19 & 36 & 11 & 14 & 5 & 6 & 6 & 12 & 1 & 2 & 4 & 2 & 4 & 4\end{array}$ $\begin{array}{lllllllllllllllllllllllllll}3 & 8 & 13 & 14 & 18 & 14 & 6 & 2 & 3 & 13 & 18 & 4 & 38 & 9 & 13 & 15 & 4 & 8 & 9 & 60 & 8 & 7 & 11 & 4\end{array}$ $\begin{array}{llllllllllllllllllllllllllll}14 & 9 & 3 & 2 & 5 & 11 & 13 & 12 & 13 & 11 & 6 & 1 & 11 & 18 & 4 & 7 & 9 & 6 & 5 & 13 & 6 & 7 & 3 & 19\end{array}$ $\begin{array}{llllllllllllllllllllllllllll}2 & 7 & 7 & 7 & 7 & 8 & 7 & 7 & 8 & 1 & 4 & 2 & 13 & 6 & 7 & 4 & 5 & 10 & 7 & 4 & 0 & 0 & 5 & 1\end{array}$ $\begin{array}{llllllllllllllllllllllllllll}8 & 3 & 3 & 2 & 0 & 0 & 2 & 5 & 1 & 5 & 1 & 7 & 10 & 3 & 17 & 7 & 4 & 4 & 8 & 10 & 1 & 0 & 0 & 0\end{array}$ $\begin{array}{lllllllllllllllllllllllllll}4 & 3 & 3 & 2 & 3 & 3 & 0 & 5 & 4 & 0 & 4 & 11 & 17 & 6 & 14 & 2 & 7 & 3 & 10 & 9 & 6 & 3 & 2 & 10\end{array}$ $\begin{array}{lllllllllllllllllllllllll}0 & 0 & 0 & 0 & 0 & 1 & 4 & 3 & 9 & 8 & 8 & 7 & 9 & 4 & 14 & 8 & 6 & 9 & 5 & 21 & 2 & 5 & 3 & 1\end{array}$ $\begin{array}{lllllllllllllllllllllllllllll}0 & 0 & 0 & 1 & 0 & 0 & 0 & 0 & 0 & 0 & 6 & 6 & 4 & 5 & 7 & 8 & 11 & 5 & 3 & 1 & 12 & 1 & 3 & 3\end{array}$ $\begin{array}{lllllllllllllllllllllllllllll}3 & 3 & 5 & 3 & 3 & 3 & 3 & 3 & 4 & 6 & 1 & 1 & 9 & 3 & 6 & 8 & 3 & 2 & 8 & 6 & 6 & 6 & 3 & 3\end{array}$ $\begin{array}{llllllllllllllllllllllllllll}0 & 0 & 0 & 0 & 0 & 0 & 0 & 0 & 0 & 0 & 0 & 0 & 1 & 7 & 7 & 4 & 10 & 8 & 3 & 7 & 3 & 4 & 10 & 1\end{array}$ $\begin{array}{llllllllllllllllllllllllll}0 & 0 & 0 & 0 & 0 & 0 & 0 & 3 & 2 & 3 & 5 & 4 & 2 & 6 & 5 & 5 & 3 & 9 & 7 & 4 & 6 & 3 & 1 & 12\end{array}$ $\begin{array}{llllllllllllllllllllllllllllll}2 & 3 & 3 & 1 & 3 & 3 & 2 & 0 & 0 & 0 & 0 & 0 & 0 & 0 & 3 & 5 & 6 & 5 & 5 & 8 & 7 & 4 & 5 & 6\end{array}$ $\begin{array}{lllllllllllllllllllllllll}3 & 3 & 0 & 0 & 0 & 0 & 1 & 0 & 0 & 0 & 0 & 0 & 0 & 0 & 0 & 4 & 0 & 2 & 6 & 0 & 2 & 4 & 6 & 9\end{array}$ $\begin{array}{lllllllllllllllllllllllllllll}0 & 1 & 3 & 2 & 3 & 4 & 3 & 3 & 2 & 0 & 1 & 3 & 5 & 4 & 2 & 0 & 3 & 2 & 6 & 10 & 7 & 5 & 1 & 0\end{array}$ 
Appendix A.--The number of $H$ and $z$ survey measurements by one-degree tessera within the conterminous 48 States--Continued

$\begin{array}{llllllllllllllllllllllllllllll}-106 & -105 & -104 & -103 & -102 & -101 & -100 & -99 & -98 & -97 & -96 & -95 & -94 & -93 & -92 & -91 & -90 & -89 & -88 & -87 & -86 & -85 & -84 & -83 & -82\end{array}$

54

52

51

50

$\begin{array}{lllllllllllllllllllllllll}13 & 16 & 14 & 18 & 30 & 26 & 20 & 39 & 32 & 30 & 9 & 10 & 3 & 12 & 29 & 8 & 13 & 18 & 5 & 8 & 11 & 1 & 0 & 6\end{array}$ $\begin{array}{lllllllllllllllllllllllllll}13 & 10 & 14 & 21 & 14 & 21 & 31 & 17 & 44 & 21 & 31 & 36 & 28 & 29 & 31 & 10 & 4 & 20 & 8 & 14 & 12 & 10 & 20 & 8\end{array}$

46

45

44

42

41

39

38

$\begin{array}{lllllllllllllllllllllllll}8 & 5 & 4 & 11 & 7 & 5 & 9 & 5 & 17 & 10 & 5 & 16 & 8 & 9 & 20 & 16 & 48 & 33 & 15 & 4 & 18 & 16 & 9 & 11\end{array}$ $\begin{array}{lllllllllllllllllllllllll}1 & 18 & 2 & 3 & 6 & 7 & 6 & 4 & 7 & 4 & 3 & 9 & 3 & 0 & 2 & 0 & 4 & 26 & 25 & 27 & 11 & 6 & 13 & 16\end{array}$ $\begin{array}{lllllllllllllllllllllllllllll}1 & 2 & 3 & 7 & 3 & 10 & 3 & 12 & 4 & 21 & 6 & 2 & 2 & 12 & 3 & 13 & 3 & 21 & 21 & 3 & 29 & 36 & 15 & 35\end{array}$ $\begin{array}{lllllllllllllllllllllllllll}0 & 1 & 3 & 3 & 5 & 4 & 5 & 7 & 3 & 5 & 10 & 9 & 9 & 15 & 14 & 4 & 18 & 9 & 10 & 5 & 2 & 31 & 13 & 6\end{array}$ $\begin{array}{llllllllllllllllllllllll}5 & 1 & 13 & 0 & 0 & 6 & 2 & 22 & 7 & 8 & 5 & 7 & 18 & 19 & 11 & 7 & 6 & 11 & 5 & 3 & 12 & 14 & 11 & 4\end{array}$ $\begin{array}{lllllllllllllllllllllllllll}2 & 4 & 4 & 5 & 8 & 6 & 7 & 9 & 8 & 8 & 15 & 12 & 28 & 14 & 23 & 7 & 16 & 14 & 11 & 8 & 6 & 10 & 19 & 16\end{array}$ $\begin{array}{lllllllllllllllllllllllllll}1 & 5 & 14 & 4 & 3 & 2 & 11 & 4 & 14 & 11 & 7 & 10 & 7 & 10 & 19 & 15 & 8 & 24 & 6 & 5 & 34 & 14 & 17 & 36\end{array}$ $\begin{array}{lllllllllllllllllllllllllll}10 & 40 & 20 & 13 & 6 & 11 & 9 & 10 & 12 & 24 & 32 & 19 & 25 & 12 & 26 & 17 & 12 & 30 & 14 & 24 & 18 & 12 & 13 & 8\end{array}$ $\begin{array}{lllllllllllllllllllllllll}14 & 14 & 15 & 23 & 19 & 19 & 22 & 23 & 23 & 27 & 22 & 25 & 21 & 21 & 28 & 19 & 16 & 29 & 19 & 37 & 26 & 22 & 20 & 26\end{array}$ $\begin{array}{llllllllllllllllllllllllll}16 & 8 & 5 & 10 & 11 & 13 & 10 & 18 & 16 & 9 & 22 & 23 & 21 & 14 & 13 & 11 & 18 & 16 & 17 & 24 & 27 & 40 & 25 & 29\end{array}$ $\begin{array}{lllllllllllllllllllllllll}16 & 19 & 3 & 13 & 9 & 8 & 6 & 8 & 39 & 11 & 69 & 9 & 15 & 19 & 15 & 24 & 20 & 19 & 31 & 41 & 41 & 37 & 49 & 40\end{array}$ $\begin{array}{llllllllllllllllllllllllll}23 & 30 & 22 & 30 & 28 & 37 & 22 & 37 & 21 & 41 & 23 & 23 & 29 & 26 & 44 & 28 & 30 & 34 & 44 & 27 & 31 & 43 & 25 & 18\end{array}$ $\begin{array}{lllllllllllllllllllllllllll}2 & 11 & 2 & 6 & 4 & 5 & 9 & 12 & 8 & 14 & 20 & 10 & 23 & 17 & 14 & 15 & 25 & 8 & 16 & 24 & 14 & 18 & 19 & 34\end{array}$ 36 35 $\begin{array}{lllllllllllllllllllllllllll}5 & 3 & 15 & 8 & 17 & 12 & 12 & 11 & 19 & 22 & 14 & 15 & 10 & 9 & 9 & 23 & 19 & 20 & 15 & 19 & 19 & 30 & 42 & 35\end{array}$ $\begin{array}{llllllllllllllllllllllllll}7 & 9 & 4 & 8 & 6 & 8 & 13 & 23 & 11 & 17 & 11 & 16 & 6 & 18 & 13 & 8 & 9 & 15 & 13 & 35 & 23 & 15 & 28 & 15\end{array}$ $\begin{array}{lllllllllllllllllllllllllll}6 & 8 & 4 & 6 & 8 & 7 & 10 & 8 & 8 & 17 & 11 & 18 & 8 & 18 & 9 & 8 & 25 & 14 & 18 & 18 & 24 & 37 & 34 & 22\end{array}$ $\begin{array}{llllllllllllllllllllllllll}10 & 5 & 5 & 5 & 15 & 12 & 13 & 9 & 13 & 11 & 9 & 7 & 15 & 15 & 13 & 20 & 11 & 18 & 18 & 20 & 12 & 15 & 20 & 16\end{array}$ $\begin{array}{llllllllllllllllllllllllll}9 & 10 & 10 & 8 & 3 & 6 & 8 & 8 & 4 & 9 & 6 & 7 & 5 & 19 & 9 & 20 & 20 & 11 & 17 & 9 & 11 & 18 & 14 & 22\end{array}$ 31

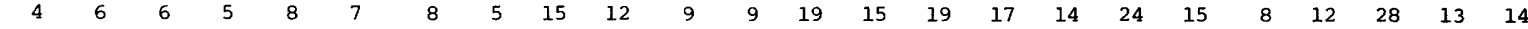
29 $\begin{array}{llllllllllllllllllllllllllllll}0 & 1 & 1 & 0 & 3 & 8 & 3 & 10 & 16 & 13 & 14 & 14 & 2 & 1 & 11 & 14 & 13 & 1 & 0 & 1 & 6 & 6 & 3 & 21\end{array}$ $\begin{array}{llllllllllllllllllllllllll}0 & 0 & 0 & 2 & 0 & 6 & 7 & 5 & 22 & 10 & 5 & 7 & 1 & 0 & 4 & 6 & 1 & 0 & 4 & 2 & 5 & 7 & 5 & 16\end{array}$ 27 26 25 24 23 22 21 20 $\begin{array}{llllllllllllllllllllllllllllll}0 & 2 & 3 & 0 & 6 & 3 & 19 & 7 & 12 & 0 & 2 & 2 & 2 & 7 & 8 & 0 & 0 & 2 & 4 & 1 & 7 & 6 & 8 & 10\end{array}$ $\begin{array}{lllllllllllllllllllllllllll}3 & 1 & 0 & 1 & 4 & 1 & 1 & 4 & 11 & 2 & 2 & 2 & 2 & 10 & 0 & 0 & 3 & 1 & 0 & 9 & 9 & 6 & 1 & 9\end{array}$ $\begin{array}{lllllllllllllllllllllllllllllllll}4 & 3 & 5 & 2 & 3 & 7 & 7 & 0 & 29 & 5 & 2 & 2 & 9 & 1 & 0 & 1 & 2 & 2 & 4 & 8 & 8 & 2 & 0 & 2\end{array}$ $\begin{array}{llllllllllllllllllllllllll}0 & 5 & 4 & 0 & 5 & 3 & 1 & 0 & 10 & 5 & 4 & 9 & 4 & 0 & 0 & 4 & 4 & 1 & 4 & 12 & 13 & 13 & 9 & 10 & \end{array}$ $\begin{array}{lllllllllllllllllllllllllllll}0 & 0 & 1 & 4 & 4 & 4 & 4 & 6 & 8 & 3 & 12 & 6 & 0 & 0 & 6 & 3 & 0 & 0 & 8 & 9 & 3 & 0 & 5 & 29\end{array}$ $\begin{array}{lllllllllllllllllllllllllll}5 & 0 & 0 & 5 & 6 & 5 & 1 & 4 & 17 & 7 & 4 & 2 & 7 & 4 & 3 & 0 & 1 & 5 & 15 & 1 & 4 & 1 & 11 & 5\end{array}$ $\begin{array}{lllllllllllllllllllllllllll}7 & 12 & 4 & 4 & 11 & 6 & 6 & 5 & 13 & 9 & 1 & 2 & 4 & 4 & 0 & 0 & 4 & 4 & 12 & 5 & 5 & 1 & 0 & 0\end{array}$ $\begin{array}{llllllllllllllllllllllllllll}1 & 3 & 12 & 13 & 15 & 14 & 17 & 11 & 16 & 16 & 14 & 7 & 7 & 12 & 5 & 3 & 15 & 11 & 9 & 17 & 4 & 4 & 1 & 0\end{array}$ 
$\begin{array}{llllllllllllllllllllllll}-82 & -81 & -80 & -79 & -78 & -77 & -76 & -75 & -74 & -73 & -72 & -71 & -70 & -69 & -68 & -67 & -66 & -65 & -64 & -63 & -62 & -61 & -60\end{array}$

$\begin{array}{llllllllllllllllllllllll}2 & 2 & 11 & 2 & 3 & 3 & 11 & 7 & 18 & 12 & 12 & 5 & 10 & 3 & 10 & 13 & 6 & 6 & 4 & 0 & 7 & 11\end{array}$

$\begin{array}{llllllllllllllllllllllllll}55 & 5 & 7 & 8 & 4 & 3 & 6 & 1 & 8 & 13 & 0 & 4 & 2 & 8 & 7 & 9 & 20 & 3 & 12 & 0 & 3 & 4 & 7\end{array}$

$\begin{array}{llllllllllllllllllllllll}54 & 2 & 2 & 8 & 3 & 2 & 7 & 6 & 12 & 20 & 3 & 7 & 13 & 9 & 4 & 9 & 5 & 0 & 11 & 2 & 4 & 6 & 16\end{array}$

$\begin{array}{llllllllllllllllllllllll}53 & 9 & 2 & 7 & 5 & 2 & 8 & 9 & 0 & 13 & 2 & 3 & 9 & 4 & 2 & 13 & 5 & 3 & 10 & 6 & 11 & 12 & 16\end{array}$

$\begin{array}{llllllllllllllllllllllllllll}52 & 6 & 8 & 9 & 6 & 7 & 18 & 5 & 5 & 17 & 7 & 3 & 9 & 12 & 10 & 10 & 15 & 0 & 13 & 7 & 4 & 8 & 9\end{array}$

$\begin{array}{llllllllllllllllllllllll}51 & 9 & 4 & 7 & 4 & 8 & 7 & 3 & 3 & 18 & 1 & 4 & 12 & 7 & 6 & 2 & 20 & 11 & 10 & 7 & 4 & 16 & 7\end{array}$

$\begin{array}{llllllllllllllllllllllllllll}50 & 22 & 10 & 12 & 9 & 5 & 10 & 5 & 12 & 12 & 8 & 9 & 4 & 5 & 13 & 16 & 20 & 7 & 6 & 0 & 12 & 4 & 3\end{array}$

$\begin{array}{lllllllllllllllllllllllll}49 & 8 & 24 & 17 & 12 & 4 & 19 & 11 & 17 & 15 & 25 & 14 & 14 & 16 & 39 & 20 & 8 & 6 & 11 & 2 & 11 & 0 & 5\end{array}$

$\begin{array}{lllllllllllllllllllllllll}48 & 13 & 6 & 38 & 2 & 2 & 8 & 3 & 15 & 13 & 12 & 0 & 27 & 26 & 32 & 16 & 19 & 7 & 9 & 11 & 10 & 15 & 8\end{array}$

$\begin{array}{llllllllllllllllllllllll}47 & 24 & 26 & 36 & 33 & 11 & 11 & 18 & 14 & 13 & 28 & 44 & 15 & 10 & 6 & 24 & 12 & 4 & 33 & 29 & 17 & 11 & 34\end{array}$

$\begin{array}{llllllllllllllllllllll}10 & 11 & 14 & 17 & 25 & 18 & 67 & 37 & 33 & 41 & 17 & 21 & 20 & 22 & 31 & 32 & 12 & 15 & 18 & 22 & 27 & 17\end{array}$

$\begin{array}{llllllllllllllllllllllll}44 & 2 & 14 & 23 & 16 & 16 & 32 & 20 & 24 & 45 & 15 & 14 & 11 & 14 & 33 & 6 & 23 & 33 & 37 & 46 & 5 & 9 & 22\end{array}$

$\begin{array}{llllllllllllllllllllll}10 & 15 & 80 & 7 & 8 & 20 & 11 & 6 & 7 & 29 & 15 & 23 & 2 & 12 & 18 & 30 & 12 & 8 & 6 & 13 & 11 & 11\end{array}$

$\begin{array}{llllllllllllllllllllllll}43 & 20 & 6 & 9 & 14 & 5 & 22 & 6 & 6 & 23 & 28 & 41 & 29 & 15 & 16 & 12 & 13 & 6 & 10 & 8 & 0 & 6 & 11\end{array}$

$\begin{array}{lllllllllllllllllllllllllll}42 & 18 & 25 & 10 & 5 & 10 & 12 & 12 & 14 & 19 & 58 & 53 & 18 & 14 & 8 & 2 & 12 & 8 & 10 & 1 & 7 & 13 & 10\end{array}$

$\begin{array}{lllllllllllllllllllllllll}41 & 16 & 17 & 13 & 6 & 11 & 31 & 28 & 55 & 34 & 18 & 9 & 5 & 4 & 6 & 5 & 7 & 2 & 9 & 4 & 0 & 9\end{array}$

$\begin{array}{rrrrrrrrrrrrrrrrrrrrrrrr}40 & 16 & 17 & 13 & 6 & 11 & 31 & 28 & 55 & 34 & 18 & 9 & 5 & 4 & 6 & 5 & 7 & 2 & 9 & 4 & 0 & 9 & 5 \\ & 39 & 35 & 34 & 24 & 34 & 24 & 71 & 27 & 5 & 2 & 5 & 2 & 7 & 3 & 7 & 8 & 0 & 2 & 3 & 10 & 3 & 6\end{array}$

$\begin{array}{llllllllllllllllllllllll}39 & 35 & 24 & 27 & 34 & 47 & 84 & 9 & 20 & 18 & 10 & 11 & 6 & 5 & 7 & 1 & 4 & 1 & 5 & 9 & 14 & 4 & 9\end{array}$

$\begin{array}{llllllllllllllllllllllllll}17 & 34 & 65 & 38 & 41 & 19 & 7 & 10 & 22 & 20 & 9 & 4 & 3 & 6 & 1 & 7 & 3 & 0 & 14 & 7 & 0 & 5\end{array}$

37

$\begin{array}{llllllllllllllllllllll}27 & 12 & 18 & 23 & 25 & 46 & 16 & 17 & 6 & 13 & 17 & 18 & 3 & 3 & 8 & 3 & 0 & 0 & 21 & 1 & 3 & 8\end{array}$

36

35

34

$\begin{array}{lllllllllllllllllllllllll}24 & 32 & 19 & 22 & 23 & 13 & 29 & 8 & 9 & 11 & 10 & 13 & 24 & 9 & 4 & 6 & 3 & 8 & 11 & 4 & 3 & 8\end{array}$ $\begin{array}{llllllllllllllllllllllll}42 & 18 & 32 & 6 & 23 & 11 & 15 & 14 & 17 & 5 & 0 & 6 & 12 & 18 & 21 & 5 & 2 & 14 & 8 & 2 & 11 & 8\end{array}$

33

$\begin{array}{lllllllllllllllllllllll}28 & 16 & 10 & 10 & 15 & 6 & 11 & 7 & 15 & 10 & 6 & 7 & 4 & 5 & 16 & 29 & 0 & 16 & 15 & 21 & 17 & 7\end{array}$

32

$\begin{array}{llllllllllllllllllllll}24 & 13 & 14 & 4 & 12 & 7 & 11 & 3 & 3 & 23 & 7 & 4 & 8 & 10 & 11 & 14 & 19 & 226 & 36 & 19 & 18 & 12\end{array}$

$\begin{array}{llllllllllllllllllllllll}15 & 2 & 8 & 8 & 11 & 11 & 6 & 9 & 0 & 8 & 12 & 3 & 0 & 3 & 7 & 0 & 11 & 12 & 10 & 10 & 11 & 8\end{array}$

31

30

$\begin{array}{llllllllllllllllllllllll}17 & 0 & 6 & 1 & 13 & 7 & 9 & 14 & 5 & 5 & 10 & 6 & 0 & 0 & 8 & 9 & 7 & 9 & 4 & 8 & 4 & 3\end{array}$

29 $\begin{array}{llllllllllllllllllllllll}29 & 9 & 3 & 0 & 0 & 14 & 4 & 9 & 6 & 10 & 6 & 6 & 7 & 0 & 11 & 5 & 7 & 4 & 4 & 1 & 7 & 4\end{array}$ $\begin{array}{llllllllllllllllllllll}10 & 33 & 3 & 1 & 0 & 5 & 8 & 10 & 7 & 4 & 13 & 13 & 7 & 8 & 11 & 0 & 11 & 11 & 2 & 3 & 4 & 4\end{array}$ $\begin{array}{llllllllllllllllllllllll}8 & 12 & 3 & 4 & 5 & 4 & 0 & 14 & 4 & 0 & 7 & 10 & 11 & 8 & 9 & 7 & 8 & 6 & 0 & 7 & 3 & 7\end{array}$ $\begin{array}{lllllllllllllllllllllllll}14 & 18 & 0 & 0 & 7 & 2 & 3 & 6 & 7 & 3 & 0 & 7 & 18 & 8 & 8 & 0 & 14 & 9 & 0 & 4 & 0 & 4\end{array}$ $\begin{array}{llllllllllllllllllllllll}10 & 11 & 2 & 0 & 7 & 6 & 5 & 7 & 2 & 11 & 0 & 6 & 3 & 19 & 6 & 14 & 13 & 5 & 7 & 4 & 0 & 1\end{array}$ $\begin{array}{llllllllllllllllllllll}40 & 6 & 4 & 0 & 4 & 2 & 9 & 0 & 8 & 6 & 11 & 0 & 3 & 9 & 8 & 8 & 14 & 14 & 8 & 9 & 1 & 0\end{array}$ $\begin{array}{lllllllllllllllllllllllllll}8 & 0 & 2 & 4 & 2 & 3 & 5 & 8 & 9 & 3 & 10 & 6 & 0 & 7 & 5 & 8 & 14 & 0 & 0 & 4 & 10 & 0\end{array}$ $\begin{array}{llllllllllllllllllllllll}1 & 10 & 6 & 2 & 3 & 2 & 4 & 5 & 13 & 4 & 3 & 28 & 26 & 4 & 11 & 9 & 16 & 6 & 10 & 5 & 10 & 5\end{array}$ $\begin{array}{llllllllllllllllllllllll}5 & 0 & 0 & 0 & 13 & 0 & 0 & 8 & 14 & 5 & 0 & 31 & 43 & 15 & 4 & 6 & 13 & 12 & 6 & 0 & 8 & 9\end{array}$ 20 $0 \quad 5 \quad 0 \quad 0$ 
Appendix B.--Residuals--U.S. repeat stations and observatories, including Alaskan repeat stations

[Station value minus model value for the date of measurement; for example, 1971.4 = 71.4; Lat, latitude; Long, longitude; Dec1, declination; Horiz, horizontal; Vert, vertical; , degrees; $\mathrm{nT}$, nanotesla]

\begin{tabular}{|c|c|c|c|c|c|c|c|c|c|}
\hline $\begin{array}{l}\text { State } \\
\text { (Abbr) }\end{array}$ & Station (name) & $\underset{0}{\text { Lat }}$ & $\underset{0}{\text { Long }}$ & $\underset{0}{\operatorname{Dec} 1}$ & $\underset{0}{\text { Dip }}$ & $\begin{array}{c}\text { Horiz } \\
\mathrm{nT}\end{array}$ & $\begin{array}{c}\text { Vert } \\
\mathrm{nT}\end{array}$ & $\begin{array}{c}\text { Tota1 } \\
\mathrm{nT}\end{array}$ & Date \\
\hline 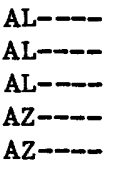 & $\begin{array}{l}\text { Marion (FISH) } \\
\text { Marion (FISH) } \\
\text { Gadsden- } \\
\text { Nogales } \\
\text { Phoenix }\end{array}$ & $\begin{array}{l}32.73 \\
32.73 \\
34.00 \\
31.35 \\
33.46\end{array}$ & $\begin{array}{r}-87.28 \\
-87.28 \\
-86.00 \\
-110.94 \\
-111.96\end{array}$ & $\begin{array}{r}-0.20 \\
-0.21 \\
0.04 \\
0.12 \\
0.33\end{array}$ & $\begin{array}{r}0.08 \\
0.09 \\
-0.14 \\
-0.09 \\
-0.05\end{array}$ & $\begin{array}{r}121 \\
98 \\
226 \\
-9 \\
-44\end{array}$ & $\begin{array}{r}421 \\
392 \\
179 \\
-164 \\
-161\end{array}$ & $\begin{aligned} & 431 \\
& 395 \\
& 258 \\
&-144 \\
&-161\end{aligned}$ & $\begin{array}{l}71.4 \\
72.9 \\
74.4 \\
72.9 \\
72.8\end{array}$ \\
\hline 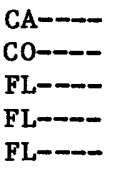 & $\begin{array}{l}\text { San Francisco (GOLF)-- } \\
\text { Cortez (ARPT) } \\
\text { Fort Meyers } \\
\text { Fort Meyers } \\
\text { Key West (RK PT) }\end{array}$ & $\begin{array}{l}37.72 \\
37.30 \\
26.61 \\
26.61 \\
24.56\end{array}$ & $\begin{array}{r}-122.49 \\
-108.63 \\
-81.88 \\
-81.88 \\
-81.68\end{array}$ & $\begin{array}{r}0.25 \\
-0.48 \\
-0.36 \\
-0.30 \\
-0.20\end{array}$ & $\begin{array}{r}-0.20 \\
0.17 \\
0.01 \\
0.07 \\
0.16\end{array}$ & $\begin{array}{r}125 \\
-201 \\
27 \\
-40 \\
-109\end{array}$ & $\begin{array}{r}-144 \\
-61 \\
61 \\
47 \\
87\end{array}$ & $\begin{array}{r}-67 \\
-142 \\
66 \\
19 \\
12\end{array}$ & $\begin{array}{l}72.7 \\
73.8 \\
71.4 \\
74.3 \\
71.3\end{array}$ \\
\hline $\begin{array}{l}\text { FL---- } \\
\text { FL---- } \\
\text { FL----- } \\
\text { FL---- } \\
\text { FL---- }\end{array}$ & $\begin{array}{l}\text { Key West (GOLF) - } \\
\text { Key West (GOLF) Aux-2- } \\
\text { Spruce Creek (TEL)--- } \\
\text { Spruce Creek (A) - } \\
\text { Spruce Creek- }\end{array}$ & $\begin{array}{l}24.57 \\
24.57 \\
29.07 \\
29.08 \\
29.07\end{array}$ & $\begin{array}{l}-81.74 \\
-81.74 \\
-81.05 \\
-81.03 \\
-81.05\end{array}$ & $\begin{array}{r}-0.19 \\
-0.18 \\
0.15 \\
0.09 \\
0.08\end{array}$ & $\begin{array}{l}0.19 \\
0.30 \\
0.54 \\
0.49 \\
0.51\end{array}$ & $\begin{array}{l}-128 \\
-236 \\
-289 \\
-263 \\
-305\end{array}$ & $\begin{array}{r}101 . \\
92 . \\
477 . \\
438 . \\
391\end{array}$ & $\begin{array}{l}13 \\
-55 \\
279 \\
258 \\
195\end{array}$ & $\begin{array}{l}71.3 \\
74.3 \\
71.4 \\
71.4 \\
74.3\end{array}$ \\
\hline $\begin{array}{l}\text { GA---- } \\
\text { GA---- } \\
\text { GA---- } \\
\text { GA---- } \\
\text { ID---- }\end{array}$ & $\begin{array}{l}\text { Bainbridge (1958) } \\
\text { Bainbridge (1958) } \\
\text { Milledgeville- } \\
\text { Waycross- } \\
\text { Weiser- }\end{array}$ & $\begin{array}{l}30.91 \\
30.91 \\
33.09 \\
31.24 \\
44.26\end{array}$ & $\begin{array}{r}-84.60 \\
-84.60 \\
-83.28 \\
-82.35 \\
-116.97\end{array}$ & $\begin{array}{r}-0.12 \\
-0.15 \\
1.11 \\
0.03 \\
0.41\end{array}$ & $\begin{array}{r}-0.13 \\
-0.14 \\
-0.04 \\
-0.14 \\
0.03\end{array}$ & $\begin{array}{r}84 . \\
56 \\
-77 \\
104 \\
98\end{array}$ & $\begin{array}{l}-105 . \\
-166 . \\
-246 . \\
-82 \\
318\end{array}$ & $\begin{array}{l}-54 \\
-121 \\
-255 \\
-25 \\
332\end{array}$ & $\begin{array}{l}71.4 \\
74.3 \\
71.4 \\
74.3 \\
73.8\end{array}$ \\
\hline $\begin{array}{l}\mathrm{ME}---- \\
\mathrm{ME}---- \\
\mathrm{ME}---- \\
\mathrm{MI}---- \\
\mathrm{MI}---\end{array}$ & $\begin{array}{l}\text { Bangor (GRIFFIN) } \\
\text { Fort Kent (B HOSP) } \\
\text { Fort Kent (PASTURE) } \\
\text { Detroit (PARK) } \\
\text { Detroit (RR) }\end{array}$ & $\begin{array}{l}44.83 \\
47.27 \\
47.25 \\
42.36 \\
42.34\end{array}$ & $\begin{array}{l}-68.81 \\
-68.60 \\
-68.59 \\
-83.26 \\
-83.25\end{array}$ & $\begin{array}{l}-0.12 \\
-0.19 \\
-0.20 \\
-0.09 \\
-0.01\end{array}$ & $\begin{array}{r}0.08 \\
0.03 \\
0.06 \\
-0.22 \\
-0.22\end{array}$ & $\begin{array}{l}-74 \\
-5 \\
-38 \\
155 \\
143\end{array}$ & $\begin{array}{r}41 . \\
91 \\
102 \\
-236 \\
-272\end{array}$ & $\begin{array}{r}17 \\
86 \\
88 \\
-178 \\
-215\end{array}$ & $\begin{array}{l}73.5 \\
73.6 \\
73.6 \\
72.5 \\
72.5\end{array}$ \\
\hline $\begin{array}{l}\text { MI---- } \\
\text { MO---- } \\
\text { MS---- } \\
\text { MS---- } \\
\text { MS---- }\end{array}$ & $\begin{array}{l}\text { Marquette (GF2 1972)-- } \\
\text { Rolla (EAST) } \\
\text { Brooklyn- } \\
\text { Grenada (A) } \\
\text { Granada (71) }\end{array}$ & $\begin{array}{l}46.54 \\
37.95 \\
31.03 \\
33.75 \\
33.75\end{array}$ & $\begin{array}{l}-87.42 \\
-91.78 \\
-89.17 \\
-89.80 \\
-89.80\end{array}$ & $\begin{array}{r}0.82 \\
-0.45 \\
-0.04 \\
-0.24 \\
-0.24\end{array}$ & $\begin{array}{r}0.01 \\
0.00 \\
-0.07 \\
0.00 \\
0.02\end{array}$ & $\begin{array}{r}-56 \\
31 \\
26 \\
72 \\
63\end{array}$ & $\begin{array}{r}-170 . \\
88 . \\
-93 . \\
160 \\
166\end{array}$ & $\begin{array}{r}-178 \\
93 \\
-70 \\
175 \\
177\end{array}$ & $\begin{array}{l}72.5 \\
73.7 \\
72.9 \\
72.9 \\
72.9\end{array}$ \\
\hline $\begin{array}{l}\mathrm{MS}---- \\
\mathrm{MT}---- \\
\mathrm{MT}---- \\
\mathrm{MT}---- \\
\mathrm{MT}----\end{array}$ & $\begin{array}{l}\text { Lamar (B GUM) } \\
\text { Billings (PP) } \\
\text { Glendive (ARPT 71) } \\
\text { Havre- } \\
\text { Helena (ARPT 71) }\end{array}$ & $\begin{array}{l}30.92 \\
45.79 \\
47.13 \\
48.56 \\
46.60\end{array}$ & $\begin{array}{r}-89.39 \\
-108.53 \\
-104.81 \\
-109.72 \\
-111.98\end{array}$ & $\begin{array}{r}0.06 \\
-0.72 \\
-0.17 \\
0.02 \\
-0.36\end{array}$ & $\begin{array}{r}-0.06 \\
-0.04 \\
0.00 \\
0.10 \\
-0.07\end{array}$ & $\begin{aligned} & 21 \\
& 81 \\
&-14 \\
&-77 \\
&-11\end{aligned}$ & $\begin{array}{l}-79 \\
113 \\
-36 \\
122 \\
-246\end{array}$ & $\begin{array}{r}-60 \\
132 \\
-38 \\
96 \\
-236\end{array}$ & $\begin{array}{l}72.9 \\
72.5 \\
71.6 \\
71.5 \\
71.6\end{array}$ \\
\hline
\end{tabular}


Appendix B.--Residuals--U.S. repeat stations and observatories, including Alaskan repeat stations --Continued.

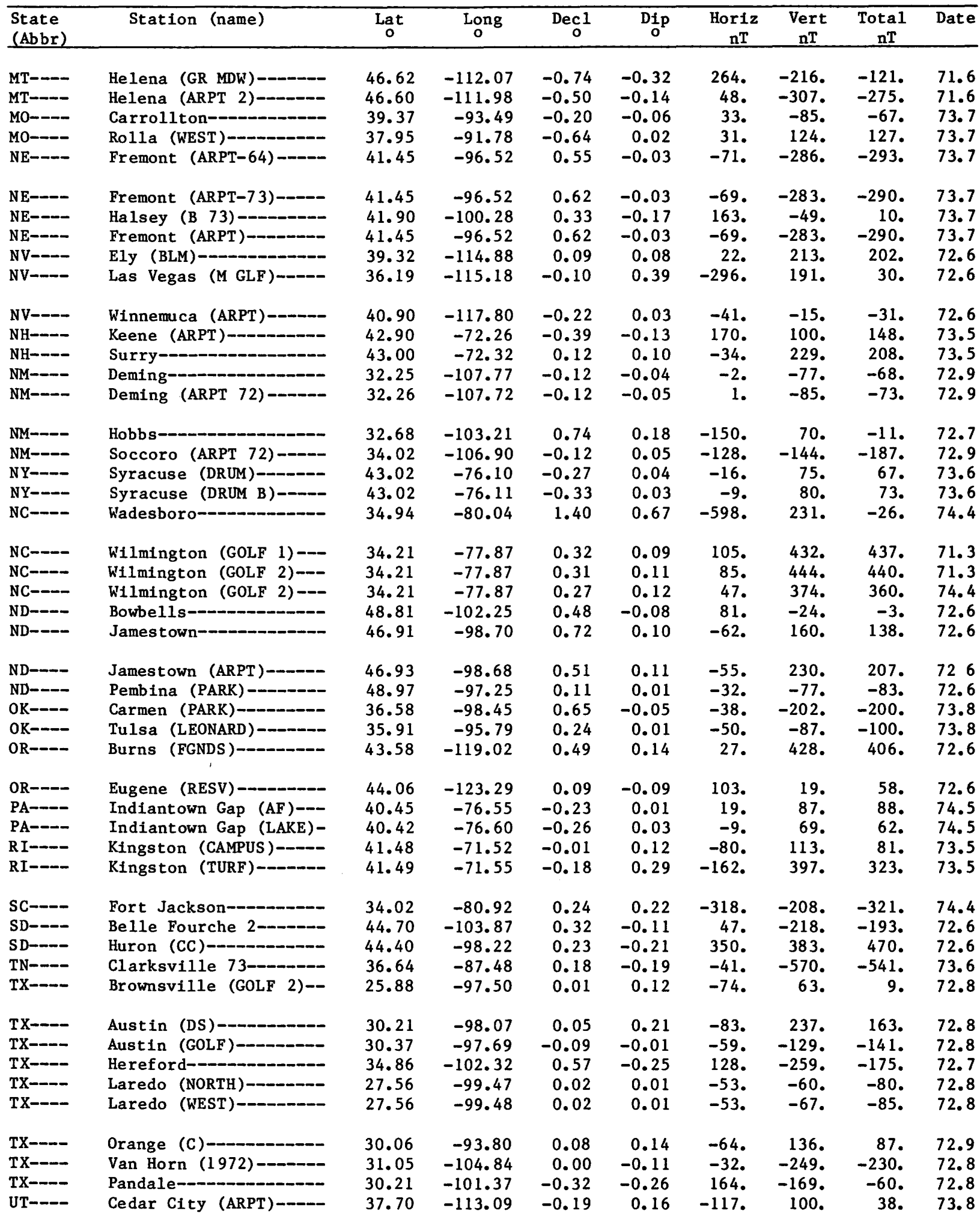


Appendix B.--Residuals.--U.S. repeat stations and observatorfes, including Alaskan repeat stations --Continued.

\begin{tabular}{|c|c|c|c|c|c|c|c|c|c|}
\hline $\begin{array}{l}\text { State } \\
\text { (Abbr) }\end{array}$ & Station (name) & $\underset{0}{\text { Lat }}$ & Long & $\begin{array}{c}\text { Decl } \\
\text { - }\end{array}$ & Dip & $\begin{array}{c}\text { Horiz } \\
\text { nT } \\
\end{array}$ & $\begin{array}{c}\text { Vert } \\
\text { nT }\end{array}$ & $\begin{array}{c}\text { Total } \\
\text { nT } \\
\end{array}$ & Date \\
\hline $\begin{array}{l}\text { UT---- } \\
\text { VT---- } \\
\text { VT---- } \\
\text { WA---- } \\
\text { WA---- }\end{array}$ & $\begin{array}{l}\text { Salt Lake City-- } \\
\text { Bur1ington (LP)- } \\
\text { Burlington (RS)- } \\
\text { Coulee Dam- } \\
\text { Quillayute- }\end{array}$ & $\begin{array}{l}40.78 \\
44.46 \\
44.47 \\
48.06 \\
47.94\end{array}$ & $\begin{array}{r}-111.86 \\
-73.16 \\
-73.20 \\
-118.95 \\
-124.55\end{array}$ & $\begin{array}{r}-0.36 \\
0.15 \\
0.13 \\
-0.01 \\
-0.04\end{array}$ & $\begin{array}{r}-0.10 \\
-0.28 \\
-0.32 \\
0.00 \\
-0.04\end{array}$ & $\begin{array}{r}75 . \\
311 . \\
353 . \\
57 . \\
40 .\end{array}$ & $\begin{aligned}-73 . \\
52 . \\
42 . \\
176 . \\
-10 .\end{aligned}$ & $\begin{array}{l}-37 . \\
141 . \\
144 . \\
184 . \\
4 .\end{array}$ & $\begin{array}{l}73.8 \\
73.6 \\
73.6 \\
71.5 \\
71.5\end{array}$ \\
\hline $\begin{array}{l}\text { WV---- } \\
\text { WA---- } \\
\text { WA--- } \\
\text { WI--- } \\
\text { WI--- }\end{array}$ & $\begin{array}{l}\text { Parkersburg (GOLF)---- } \\
\text { Seattle (ST EDWD)--- } \\
\text { Seattle (ST THOS)- } \\
\text { Tomahawk } \\
\text { Tomahawk (ARPT) } \\
\end{array}$ & $\begin{array}{l}39.28 \\
47.73 \\
47.73 \\
45.47 \\
45.47\end{array}$ & $\begin{array}{r}-81.51 \\
-122.26 \\
-122.26 \\
-89.74 \\
-89.74\end{array}$ & $\begin{array}{r}0.16 \\
0.05 \\
-0.02 \\
0.49 \\
0.01\end{array}$ & $\begin{array}{r}0.15 \\
0.01 \\
0.03 \\
-0.18 \\
-0.33\end{array}$ & $\begin{array}{l}-28 . \\
-2 . \\
-52 \\
110 \\
265\end{array}$ & $\begin{array}{r}361 . \\
35 . \\
-47 . \\
-316 . \\
-305\end{array}$ & $\begin{array}{r}330 . \\
33 . \\
-62 . \\
-274 . \\
-221 .\end{array}$ & $\begin{array}{l}72.4 \\
72.6 \\
72.6 \\
72.6 \\
72.6\end{array}$ \\
\hline $\begin{array}{l}\text { WI--- } \\
\text { WY--- } \\
\text { WA--- } \\
\text { CO--- } \\
\text { VA--- }\end{array}$ & $\begin{array}{l}\text { Eau Claire--_-_-_- } \\
\text { Sheridan } \\
\text { Newport } \\
\text { Boulder } \\
\text { Fredericksburg }\end{array}$ & $\begin{array}{l}44.83 \\
44.85 \\
48.26 \\
40.14 \\
38.20\end{array}$ & $\begin{array}{r}-91.53 \\
-106.96 \\
-117.12 \\
-105.24 \\
-77.37\end{array}$ & $\begin{array}{r}0.74 \\
-0.24 \\
0.12 \\
0.17 \\
-0.19\end{array}$ & $\begin{array}{r}-0.20 \\
-0.08 \\
0.01 \\
-0.11 \\
0.04\end{array}$ & $\begin{array}{r}94 . \\
18 . \\
1 . \\
20 . \\
47 .\end{array}$ & $\begin{array}{r}-404 . \\
-187 . \\
33 . \\
-224 . \\
222 .\end{array}$ & $\begin{array}{r}-362 . \\
-171 . \\
32 . \\
-200 . \\
224 .\end{array}$ & $\begin{array}{l}72.6 \\
71.6 \\
74.5 \\
74.5 \\
74.5\end{array}$ \\
\hline $\begin{array}{l}\text { CA---- } \\
\text { TX---- } \\
\text { AZ---- } \\
\text { AK---- } \\
\text { AK--- }\end{array}$ & $\begin{array}{l}\text { Castle Rock }{ }^{1}- \\
\text { Dallas } \\
\text { Tucson } \\
\text { Anchorage (NBS) } \\
\text { Barter Is (IGY)- }\end{array}$ & $\begin{array}{l}37.24 \\
32.99 \\
32.25 \\
61.24 \\
70.13\end{array}$ & $\begin{array}{r}-122.13 \\
-96.75 \\
-110.83 \\
-149.87 \\
-143.65\end{array}$ & $\begin{array}{r}0.04 \\
0.41 \\
-0.12 \\
0.63 \\
0.40\end{array}$ & $\begin{array}{r}0.17 \\
-0.13 \\
0.20 \\
0.18 \\
0.07\end{array}$ & $\begin{array}{r}-123 . \\
17 . \\
-109 . \\
-21 . \\
-85 .\end{array}$ & $\begin{array}{r}83 . \\
-220 . \\
166 . \\
565 \\
-77\end{array}$ & $\begin{array}{r}15 . \\
-188 . \\
87 . \\
537 . \\
-89 .\end{array}$ & $\begin{array}{l}74.5 \\
74.5 \\
74.5 \\
75.4 \\
75.6\end{array}$ \\
\hline $\begin{array}{l}\text { AK---- } \\
\text { AK---- } \\
\text { AK---- } \\
\text { AK---- } \\
\text { AK--- }\end{array}$ & $\begin{array}{l}\text { Bethel (AIRPORT) } \\
\text { Chitina- } \\
\text { Cordova } 3 \text { (1975)- } \\
\text { Fort Yukon (IGY)- } \\
\text { Homer } 1 \text { (AIRPORT) }\end{array}$ & $\begin{array}{l}60.78 \\
61.52 \\
60.55 \\
66.56 \\
59.64\end{array}$ & $\begin{array}{l}-161.83 \\
-144.44 \\
-145.76 \\
-145.26 \\
-151.49\end{array}$ & $\begin{array}{r}0.40 \\
0.66 \\
-0.07 \\
-0.03 \\
-0.81\end{array}$ & $\begin{array}{r}0.07 \\
-0.13 \\
-0.08 \\
0.10 \\
-0.07\end{array}$ & $\begin{array}{r}9 . \\
102 . \\
103 . \\
-112 . \\
73 .\end{array}$ & $\begin{array}{r}241 . \\
-116 . \\
82 . \\
-56 . \\
2 .\end{array}$ & $\begin{array}{l}231 . \\
-85 . \\
108 . \\
-78 . \\
24 .\end{array}$ & $\begin{array}{l}75.7 \\
75.5 \\
75.5 \\
75.6 \\
75.4\end{array}$ \\
\hline $\begin{array}{l}\text { AK---- } \\
\text { AK---- } \\
\text { AK---- } \\
\text { AK---- } \\
\text { AK---- }\end{array}$ & $\begin{array}{l}\text { Hughes (1975)-------- } \\
\text { Kenai (APT) 1975----- } \\
\text { Kodiak (1975)-- } \\
\text { Kotzebue (IGY)-- } \\
\text { Kotzebue (1975)--- }\end{array}$ & $\begin{array}{l}66.05 \\
60.56 \\
57.80 \\
66.88 \\
66.88\end{array}$ & $\begin{array}{l}-154.26 \\
-151.26 \\
-152.37 \\
-162.64 \\
-162.64\end{array}$ & $\begin{array}{l}0.91 \\
0.32 \\
0.26 \\
0.40 \\
0.33\end{array}$ & $\begin{array}{r}0.15 \\
0.19 \\
-0.02 \\
-0.09 \\
-0.03\end{array}$ & $\begin{array}{r}-198 . \\
-48 . \\
47 . \\
62 . \\
8 .\end{array}$ & $\begin{array}{r}-209 . \\
480 . \\
80 . \\
-119 . \\
-88 .\end{array}$ & $\begin{array}{r}-249 . \\
445 . \\
91 . \\
-101 . \\
-84 .\end{array}$ & $\begin{array}{l}75.7 \\
75.4 \\
75.5 \\
75.6 \\
75.6\end{array}$ \\
\hline $\begin{array}{l}\text { AK---- } \\
\text { AK---- } \\
\text { AK---- } \\
\text { AK---- } \\
\text { Ak---- }\end{array}$ & $\begin{array}{l}\text { Nome (APT) Aux A (58)- } \\
\text { Northway (IGY) 2- } \\
\text { Platinum- } \\
\text { Pt Hope (CEMETERY)-- } \\
\text { Prudhoe Bay (1975)--- }\end{array}$ & $\begin{array}{l}64.53 \\
63.02 \\
59.02 \\
68.35 \\
70.29\end{array}$ & $\begin{array}{l}-165.37 \\
-141.80 \\
-161.82 \\
-166.78 \\
-148.70\end{array}$ & $\begin{array}{r}0.19 \\
0.21 \\
-0.67 \\
0.71 \\
0.46\end{array}$ & $\begin{array}{r}-0.02 \\
0.17 \\
0.32 \\
-0.12 \\
0.05\end{array}$ & $\begin{array}{l}-20 \\
-131 \\
-301 \\
114 \\
-73\end{array}$ & $\begin{aligned}-129 . \\
163 . \\
47 . \\
-40 . \\
-123 .\end{aligned}$ & $\begin{array}{l}-130 . \\
128 . \\
-56 . \\
-14 \\
-134\end{array}$ & $\begin{array}{l}75.6 \\
75.5 \\
75.7 \\
75.6 \\
75.6\end{array}$ \\
\hline $\begin{array}{l}\text { AK---- } \\
\text { AK---- } \\
\text { AK---- } \\
\text { AK---- } \\
\text { AK---- }\end{array}$ & $\begin{array}{l}\text { Ruby } 3 \text { (1975)- } \\
\text { Seward- } \\
\text { Shungnak- } \\
\text { Unalakleet (1975) } \\
\text { Unalaska- }\end{array}$ & $\begin{array}{l}64.73 \\
60.13 \\
66.89 \\
63.89 \\
53.86\end{array}$ & $\begin{array}{l}-155.46 \\
-149.42 \\
-157.03 \\
-160.80 \\
-166.55\end{array}$ & $\begin{array}{l}0.19 \\
0.60 \\
0.31 \\
0.57 \\
1.22\end{array}$ & $\begin{array}{r}-0.06 \\
-0.03 \\
-0.05 \\
0.04 \\
-0.28\end{array}$ & $\begin{array}{r}46 . \\
68 . \\
63 . \\
-25 . \\
152 .\end{array}$ & $\begin{array}{r}-69 . \\
115 . \\
38 . \\
45 . \\
-266 .\end{array}$ & $\begin{array}{r}-55 . \\
130 . \\
51 . \\
36 . \\
-179 .\end{array}$ & $\begin{array}{l}75.6 \\
75.5 \\
75.6 \\
75.7 \\
75.7\end{array}$ \\
\hline $\begin{array}{l}\text { AK---- } \\
\text { AK---- } \\
\text { AK---- } \\
\text { AK---- }\end{array}$ & $\begin{array}{l}\text { Yakutaf } 5 \text { (1975)--- } \\
\text { Barrow } 1 \text { - } \\
\text { Colleqe } \\
\text { Sitka }\end{array}$ & $\begin{array}{l}59.51 \\
71.30 \\
64.86 \\
57.06\end{array}$ & $\begin{array}{l}-139.66 \\
-156.75 \\
-147.84 \\
-135.33\end{array}$ & $\begin{array}{r}-0.21 \\
0.46 \\
0.26 \\
0.13\end{array}$ & $\begin{array}{r}0.16 \\
-0.07 \\
0.06 \\
-0.08\end{array}$ & $\begin{array}{r}-162 . \\
49 . \\
-67 . \\
63 .\end{array}$ & $\begin{array}{l}-1 \\
-116 \\
-25 \\
-67\end{array}$ & $\begin{array}{l}-43 \\
-106 \\
-40 \\
-47\end{array}$ & $\begin{array}{l}75.5 \\
74.5 \\
74.5 \\
74.5\end{array}$ \\
\hline
\end{tabular}

10bservatory. 
[The coeficicients can be related to the function as follows: The coefficients numbered $1,2,3,4,5,6, \ldots .35,36$ are equivalent to the coefficients $a_{00}, a_{10}, a_{20}, a_{21}, a_{22}, \ldots a_{77}$, of the function]

\begin{tabular}{|c|c|c|c|c|c|}
\hline \multicolumn{2}{|c|}{$\begin{array}{c}\text { Declination for band } \\
1 \text { in conterminous } \\
48 \text { States }\end{array}$} & \multicolumn{2}{|c|}{$\begin{array}{c}\text { Declination for band } \\
2 \text { in conterminous } \\
48 \text { States }\end{array}$} & \multicolumn{2}{|c|}{$\begin{array}{c}\text { Declination for band } \\
3 \text { in conterminous } \\
48 \text { States }\end{array}$} \\
\hline $\begin{array}{r}-1.28334 e+001 \\
5.01082 \mathrm{e}-001 \\
-7.83442 \mathrm{e}-001 \\
-1.27714 \mathrm{e}-002 \\
4.01399 \mathrm{e}-004\end{array}$ & $\begin{array}{l}1 \\
2 \\
3 \\
4 \\
5\end{array}$ & $\begin{array}{r}-2.67351 \mathrm{e}+000 \\
2.93044 \mathrm{e}-001 \\
-8.34178 \mathrm{e}-001 \\
-1.93867 \mathrm{e}-002 \\
2.36374 \mathrm{e}-002\end{array}$ & $\begin{array}{l}1 \\
2 \\
3 \\
4 \\
5\end{array}$ & $\begin{array}{r}6.75344 e+000 \\
-2.61824 e-002 \\
-6.07717 e-001 \\
-1.48153 e-002 \\
3.12457 e-002\end{array}$ & $\begin{array}{l}1 \\
2 \\
3 \\
4 \\
5\end{array}$ \\
\hline $\begin{array}{r}2.24954 e-002 \\
3.13086 e-004 \\
4.48060 e-004 \\
-1.10156 e-003 \\
9.44875 e-004\end{array}$ & $\begin{array}{r}6 \\
7 \\
8 \\
9 \\
10\end{array}$ & $\begin{array}{r}1.12795 e-002 \\
1.57874 e-003 \\
-2.94205 e-004 \\
-1.02440 e-003 \\
-2.44400 e-003\end{array}$ & $\begin{array}{r}6 \\
7 \\
8 \\
9 \\
10\end{array}$ & $\begin{array}{r}-1.00242 \mathrm{e}-002 \\
1.84189 \mathrm{e}-003 \\
-1.49579 \mathrm{e}-003 \\
1.43483 \mathrm{e}-003 \\
-9.51459 \mathrm{e}-004\end{array}$ & $\begin{array}{r}6 \\
7 \\
8 \\
9 \\
10\end{array}$ \\
\hline $\begin{array}{r}9.11331 \mathrm{e}-006 \\
5.93444 \mathrm{e}-005 \\
-3.52190 \mathrm{e}-006 \\
7.38989 \mathrm{e}-005 \\
-3.28303 \mathrm{e}-004\end{array}$ & $\begin{array}{l}11 \\
12 \\
13 \\
14 \\
15\end{array}$ & $\begin{array}{r}1.65647 e-004 \\
1.50505 e-005 \\
1.24418 e-005 \\
-1.10751 e-004 \\
-3.42778 e-004\end{array}$ & $\begin{array}{l}11 \\
12 \\
13 \\
14 \\
15\end{array}$ & $\begin{array}{r}1.61507 e-004 \\
-1.15636 e-004 \\
7.82886 e-005 \\
2.73149 e-005 \\
-4.35186 e-005\end{array}$ & $\begin{array}{l}11 \\
12 \\
13 \\
14 \\
15\end{array}$ \\
\hline $\begin{array}{r}6.01171 e-007 \\
-5.53779 e-006 \\
-1.60637 e-006 \\
-2.94657 e-006 \\
4.97808 e-006\end{array}$ & $\begin{array}{l}16 \\
17 \\
18 \\
19 \\
20\end{array}$ & $\begin{array}{r}-1.31804 e-005 \\
-7.94759 e-006 \\
-1.91323 e-005 \\
1.87905 e-005 \\
4.95508 e-005\end{array}$ & $\begin{array}{l}16 \\
17 \\
18 \\
19 \\
20\end{array}$ & $\begin{array}{r}-1.72357 \mathrm{e}-005 \\
1.51265 \mathrm{e}-005 \\
-1.97283 \mathrm{e}-005 \\
-3.45291 \mathrm{e}-006 \\
-1.24430 \mathrm{e}-005\end{array}$ & $\begin{array}{l}16 \\
17 \\
18 \\
19 \\
20\end{array}$ \\
\hline $\begin{array}{r}-7.12612 e-006 \\
-5.90044 e-008 \\
-3.61589 e-007 \\
1.30831 e-007 \\
-5.39791 e-008\end{array}$ & $\begin{array}{l}21 \\
22 \\
23 \\
24 \\
25\end{array}$ & $\begin{array}{r}5.29946 e-005 \\
-7.03108 \mathrm{e}-007 \\
2.07349 \mathrm{e}-007 \\
6.24651 \mathrm{e}-008 \\
-3.95413 \mathrm{e}-007\end{array}$ & $\begin{array}{l}21 \\
22 \\
23 \\
24 \\
25\end{array}$ & $\begin{array}{r}1.21007 \mathrm{e}-005 \\
-4.98975 \mathrm{e}-007 \\
5.00559 \mathrm{e}-007 \\
-4.41287 \mathrm{e}-007 \\
5.95931 \mathrm{e}-007\end{array}$ & $\begin{array}{l}21 \\
22 \\
23 \\
24 \\
25\end{array}$ \\
\hline $\begin{array}{r}-3.82329 \mathrm{e}-008 \\
-4.19099 \mathrm{e}-007 \\
2.71842 \mathrm{e}-006 \\
-1.59998 \mathrm{e}-010 \\
2.66724 \mathrm{e}-008\end{array}$ & $\begin{array}{l}26 \\
27 \\
28 \\
29 \\
30\end{array}$ & $\begin{array}{r}-8.15560 \mathrm{e}-008 \\
1.19152 \mathrm{e}-006 \\
2.62015 \mathrm{e}-006 \\
4.77462 \mathrm{e}-008 \\
1.87578 \mathrm{e}-008\end{array}$ & $\begin{array}{l}26 \\
27 \\
28 \\
29 \\
30\end{array}$ & $\begin{array}{r}-1.80810 \mathrm{e}-007 \\
-1.25743 \mathrm{e}-006 \\
4.17432 \mathrm{e}-007 \\
4.68736 \mathrm{e}-008 \\
-5.95585 \mathrm{e}-008\end{array}$ & $\begin{array}{l}26 \\
27 \\
28 \\
29 \\
30\end{array}$ \\
\hline $\begin{array}{r}-1.07483 \mathrm{e}-008 \\
-1.20473 \mathrm{e}-008 \\
3.34444 \mathrm{e}-008 \\
3.90318 \mathrm{e}-008 \\
-3.19948 \mathrm{e}-008 \\
3.55637 \mathrm{e}-008\end{array}$ & $\begin{array}{l}31 \\
32 \\
33 \\
34 \\
35 \\
36\end{array}$ & $\begin{array}{r}4.05456 e-008 \\
-1.07154 e-008 \\
5.66433 e-008 \\
-1.53129 e-007 \\
-4.16239 e-007 \\
-2.64805 e-007\end{array}$ & $\begin{array}{l}31 \\
32 \\
33 \\
34 \\
35 \\
36\end{array}$ & $\begin{array}{r}7.54625 e-008 \\
-2.59804 \mathrm{e}-008 \\
7.19575 \mathrm{e}-008 \\
9.23312 \mathrm{e}-008 \\
-4.68828 \mathrm{e}-009 \\
-7.84304 \mathrm{e}-008\end{array}$ & $\begin{array}{l}31 \\
32 \\
33 \\
34 \\
35 \\
36\end{array}$ \\
\hline
\end{tabular}

\begin{tabular}{|c|c|c|c|c|c|}
\hline \multicolumn{2}{|c|}{$\begin{array}{c}\text { Declination for band } \\
4 \text { in conterminous } \\
48 \text { States }\end{array}$} & \multicolumn{2}{|c|}{$\begin{array}{c}\text { Declination for band } \\
5 \text { in conterminous } \\
48 \text { States } \\
\end{array}$} & \multicolumn{2}{|c|}{$\begin{array}{c}\text { Secular change in } \\
\text { conterminous } \\
48 \text { States } \\
\end{array}$} \\
\hline $\begin{array}{r}1.30355 \mathrm{e}+001 \\
-2.64830 \mathrm{e}-001 \\
-4.03658 \mathrm{e}-001 \\
-9.50477 \mathrm{e}-004 \\
1.73702 \mathrm{e}-002\end{array}$ & $\begin{array}{l}1 \\
2 \\
3 \\
4 \\
5\end{array}$ & $\begin{array}{r}1.62869 \mathrm{e}+001 \\
-4.17347 \mathrm{e}-001 \\
-1.79067 \mathrm{e}-001 \\
6.25598 \mathrm{e}-003 \\
1.14608 \mathrm{e}-002\end{array}$ & $\begin{array}{l}1 \\
2 \\
3 \\
4 \\
5\end{array}$ & $\begin{array}{r}-5.91190 \mathrm{e}+000 \\
-1.68142 \mathrm{e}-001 \\
-6.48094 \mathrm{e}-002 \\
5.93866 \mathrm{e}-003 \\
-2.44053 \mathrm{e}-002\end{array}$ & $\begin{array}{l}1 \\
2 \\
3 \\
4 \\
5\end{array}$ \\
\hline $\begin{array}{r}-1.41128 \mathrm{e}-002 \\
4.06045 \mathrm{e}-004 \\
-6.43100 \mathrm{e}-004 \\
4.28903 \mathrm{e}-004 \\
2.70990 \mathrm{e}-004\end{array}$ & $\begin{array}{r}6 \\
7 \\
8 \\
9 \\
10\end{array}$ & $\begin{array}{r}-7.77271 \mathrm{e}-003 \\
1.43361 \mathrm{e}-004 \\
2.79125 \mathrm{e}-004 \\
1.57742 \mathrm{e}-004 \\
1.65545 \mathrm{e}-004\end{array}$ & $\begin{array}{r}6 \\
7 \\
8 \\
9 \\
10\end{array}$ & $\begin{array}{r}5.85988 e-003 \\
-3.50743 e-004 \\
3.53160 e-004 \\
-6.38998 e-005 \\
2.92968 e-004\end{array}$ & $\begin{array}{r}6 \\
7 \\
8 \\
9 \\
10\end{array}$ \\
\hline $\begin{array}{l}5.78007 e-005 \\
1.63057 e-005 \\
3.03403 e-005 \\
5.68502 e-006 \\
1.40600 e-005\end{array}$ & $\begin{array}{l}11 \\
12 \\
13 \\
14 \\
15\end{array}$ & $\begin{array}{r}4.11723 e-006 \\
-7.78303 e-005 \\
7.57472 e-005 \\
-7.25254 e-005 \\
-5.68903 e-005\end{array}$ & $\begin{array}{l}11 \\
12 \\
13 \\
14 \\
15\end{array}$ & $\begin{array}{r}3.15289 \mathrm{e}-005 \\
5.54197 \mathrm{e}-005 \\
-9.22212 \mathrm{e}-006 \\
1.83674 \mathrm{e}-005 \\
3.60506 \mathrm{e}-006\end{array}$ & $\begin{array}{l}11 \\
12 \\
13 \\
14 \\
15\end{array}$ \\
\hline $\begin{array}{r}-4.28104 e-006 \\
3.08059 e-006 \\
-2.19284 e-006 \\
3.48935 e-006 \\
2.15235 e-006\end{array}$ & $\begin{array}{l}16 \\
17 \\
18 \\
19 \\
20\end{array}$ & $\begin{array}{r}-2.37039 e-006 \\
1.50295 e-006 \\
8.47179 e-006 \\
-2.91265 e-005 \\
1.40922 e-005\end{array}$ & $\begin{array}{l}16 \\
17 \\
18 \\
19 \\
20\end{array}$ & $\begin{array}{r}9.04572 e-007 \\
3.37064 e-007 \\
1.88045 e-007 \\
-3.41158 e-007 \\
3.90881 e-008\end{array}$ & $\begin{array}{l}16 \\
17 \\
18 \\
19 \\
20\end{array}$ \\
\hline $\begin{array}{l}-1.37227 \mathrm{e}-005 \\
-1.43236 \mathrm{e}-007 \\
-3.37714 \mathrm{e}-008 \\
-1.65453 \mathrm{e}-007 \\
-1.64965 \mathrm{e}-008\end{array}$ & $\begin{array}{l}21 \\
22 \\
23 \\
24 \\
25\end{array}$ & $\begin{array}{r}1.76083 e-006 \\
-2.33909 e-008 \\
2.62577 e-007 \\
-4.61616 e-007 \\
7.10883 e-007\end{array}$ & $\begin{array}{l}21 \\
22 \\
23 \\
24 \\
25\end{array}$ & $\begin{array}{r}-1.23375 e-007 \\
-9.49271 \mathrm{e}-008 \\
-1.11951 \mathrm{e}-007 \\
1.23254 \mathrm{e}-008 \\
-2.28627 \mathrm{e}-008\end{array}$ & $\begin{array}{l}21 \\
22 \\
23 \\
24 \\
25\end{array}$ \\
\hline $\begin{array}{r}-2.04933 e-007 \\
9.80213 e-008 \\
2.92552 e-007 \\
9.88492 e-009 \\
-9.18516 e-009\end{array}$ & $\begin{array}{l}26 \\
27 \\
28 \\
29 \\
30\end{array}$ & $\begin{array}{r}1.69253 e-007 \\
-1.26358 \mathrm{e}-006 \\
1.47596 e-006 \\
6.27482 \mathrm{e}-009 \\
-8.55351 \mathrm{e}-009\end{array}$ & $\begin{array}{l}26 \\
27 \\
28 \\
29 \\
30\end{array}$ & $\begin{array}{l}-4.11474 e-009 \\
-4.37217 e-009 \\
-2.49154 e-009\end{array}$ & $\begin{array}{l}26 \\
27 \\
28\end{array}$ \\
\hline $\begin{array}{r}5.95536 e-009 \\
1.05229 e-008 \\
1.37968 e-008 \\
-6.92023 e-008 \\
-1.36467 e-008 \\
1.52980 e-007\end{array}$ & $\begin{array}{l}31 \\
32 \\
33 \\
34 \\
35 \\
36\end{array}$ & $\begin{array}{r}-4.70189 e-008 \\
1.34212 e-007 \\
-2.76226 e-008 \\
-4.80626 e-008 \\
2.37465 e-008 \\
-8.59178 e-009\end{array}$ & $\begin{array}{l}31 \\
32 \\
33 \\
34 \\
35 \\
36\end{array}$ & & \\
\hline
\end{tabular}

Vertical intensity for

conterminous 48 States
Secular change in horizontal intensity for 48 States
Secular change in vertical intensity for 48 States

$\begin{array}{rr}1.11283 e+004 & 1 \\ 5.93553 e+002 & 2 \\ -9.76034 \mathrm{e}+001 & 3 \\ -7.37877 \mathrm{e}+000 & 4 \\ -1.39807 \mathrm{e}-001 & 5 \\ & \\ 1.76988 \mathrm{e}+000 & 6 \\ -1.23084 \mathrm{e}-001 & 7 \\ 1.06707 \mathrm{e}-001 & 8 \\ -2.53787 \mathrm{e}-001 & 9 \\ 6.94785 \mathrm{e}-002 & 10 \\ & \\ 1.01022 \mathrm{e}-002 & 11 \\ -4.45213 \mathrm{e}-003 & 12 \\ 2.05698 \mathrm{e}-003 & 13 \\ 8.49875 \mathrm{e}-004 & 14 \\ 4.39136 \mathrm{e}-004 & 15 \\ & \\ 3.38103 e-004 & 16 \\ -7.43830 \mathrm{e}-005 & 17 \\ 9.43317 \mathrm{e}-005 & 18 \\ -9.52152 \mathrm{e}-005 & 19 \\ 1.18625 \mathrm{e}-004 & 20\end{array}$

$\begin{array}{rc}-4.92413 \mathrm{e}-006 & 21 \\ -2.07456 \mathrm{e}-005 & 22 \\ 5.69064 \mathrm{e}-006 & 23 \\ -5.69877 \mathrm{e}-007 & 24 \\ 1.41786 \mathrm{e}-006 & 25 \\ & \\ -1.77985 \mathrm{e}-006 & 26 \\ -3.64791 \mathrm{e}-007 & 27 \\ -4.97382 \mathrm{e}-007 & 28 \\ -7.86331 \mathrm{e}-007 & 29 \\ -6.84156 \mathrm{e}-008 & 30 \\ & \\ -3.28319 \mathrm{e}-008 & 31 \\ 1.00894 \mathrm{e}-007 & 32 \\ 7.58462 \mathrm{e}-009 & 33 \\ -2.27681 \mathrm{e}-009 & 34 \\ -3.43554 \mathrm{e}-008 & 35 \\ -9.48324 \mathrm{e}-009 & 36\end{array}$

$\begin{array}{rr}2.22299 \mathrm{e}+004 & 1 \\ -8.05063 \mathrm{e}+002 & 2 \\ 8.25874 \mathrm{e}+001 & 3 \\ -1.65495 \mathrm{e}+001 & 4 \\ 5.82352 \mathrm{e}+000 & 5 \\ & \\ -7.58918 \mathrm{e}+000 & 6 \\ 1.65418 \mathrm{e}-001 & 7 \\ -1.30201 \mathrm{e}-001 & 8 \\ 3.81803 \mathrm{e}-002 & 9 \\ -4.28637 \mathrm{e}-002 & 10 \\ & \\ 3.09667 \mathrm{e}-003 & 11 \\ 4.57084 \mathrm{e}-004 & 12 \\ 1.13402 \mathrm{e}-002 & 13 \\ -4.59439 \mathrm{e}-003 & 14 \\ 1.79758 \mathrm{e}-003 & 15 \\ & \\ -1.74924 \mathrm{e}-004 & 16 \\ 5.25474 \mathrm{e}-004 & 17 \\ 3.66931 \mathrm{e}-005 & 18 \\ 2.66568 \mathrm{e}-005 & 19 \\ -5.80020 \mathrm{e}-005 & 20\end{array}$

$\begin{array}{rr}-9.27872 e-006 & 21 \\ 4.24327 e-006 & 22 \\ -2.58297 e-006 & 23 \\ -8.01126 e-006 & 24 \\ 1.65673 e-006 & 25 \\ & \\ -3.03678 e-006 & 26 \\ 1.24441 \mathrm{e}-006 & 27 \\ -3.95024 \mathrm{e}-007 & 28 \\ 3.37905 \mathrm{e}-007 & 29 \\ -6.94873 \mathrm{e}-007 & 30 \\ & \\ -1.32518 \mathrm{e}-007 & 31 \\ -2.68581 \mathrm{e}-007 & 32 \\ 3.81435 \mathrm{e}-008 & 33 \\ 4.44719 \mathrm{e}-008 & 34 \\ 1.62086 \mathrm{e}-008 & 35 \\ 5.55452 \mathrm{e}-009 & 36\end{array}$
$-3.70315 e+000$ $2.63540 \mathrm{e}+000 \quad 3$ $\begin{array}{ll}-1.61374 e-001 & 4 \\ -2.14846 e-002 & 5\end{array}$

4.01055e-002 6 $7.56505 \mathrm{e}-003$ $-5.45952 \mathrm{e}-003$ $2.64150 \mathrm{e}-0039$ $-1.10277 \mathrm{e}-00310$

9.34116e-004 11 $9.15822 \mathrm{e}-005 \quad 12$ $9.15822 e-005$
$-4.94533 e-005$
13 $\begin{array}{rr}-4.94533 e-005 & 13 \\ 5.47427 e-005 & 14\end{array}$ $-2.62497 \mathrm{e}-00515$

$-6.85746 e-006 \quad 16$ $-1.38716 \mathrm{e}-007 \quad 17$ $-4.11077 \mathrm{e}-006 \quad 18$ $\begin{array}{rr}-4.11077 \mathrm{e}-006 & 18 \\ 1.84179 \mathrm{e}-006 & 19\end{array}$ $-6.31355 e-00720$

$1.70413 \mathrm{e}-007 \quad 21$ $-2.05289 e-006 \quad 22$ $-1.61247 \mathrm{e}-00823$ $-1.91784 \mathrm{e}-007 \quad 24$ $-8.08162 \mathrm{e}-00825$

4.97069e-008 26 $-2.27589 e-008 \quad 27$ $3.80636 \mathrm{e}-00928$
$-5.37212 \mathrm{e}+001$ -4.10167 e+000 $-1.70578 \mathrm{e}+000$ 2.71050e-001 $-1.65244 \mathrm{e}-001$

$-7.91053 \mathrm{e}-002$ $5.79294 \mathrm{e}-0037$ $2.38760 \mathrm{e}-0038$ $-3.30138 e-004$ 8.04257e-004 10

$-1.13355 \mathrm{e}-003 \quad 11$ $5.58794 \mathrm{e}-005 \quad 12$ $-1.78677 \mathrm{e}-00513$ $-1.95663 \mathrm{e}-00514$ $1.10482 \mathrm{e}-004 \quad 15$

$-6.10629 e-00616$ -4. $91566 e-006 \quad 17$ -2.97886e-006 18 $-6.65628 \mathrm{e}-00719$ $1.59898 \mathrm{e}-00720$

$-8.00258 e-00821$ $2.05089 e-006 \quad 22$ 2. $94269 e-007 \quad 23$ 3. $37098 \mathrm{e}-007 \quad 24$ 4. 86363e-008 25

$-1.26056 \mathrm{e}-00726$ $5.20045 e-00827$ -3.8345 le-008 28 
Appendix C.--Coefficients of chart models--Continued

\begin{tabular}{|c|c|c|c|c|c|c|c|c|c|c|c|}
\hline $\begin{array}{c}\begin{array}{c}\text { Declination } \\
\text { Alaska }\end{array} \\
\end{array}$ & $\begin{array}{r}\text { for } \\
1\end{array}$ & \multicolumn{2}{|c|}{$\begin{array}{l}\text { Horizontal inten- } \\
\text { sity for Alaska }\end{array}$} & \multicolumn{2}{|c|}{$\begin{array}{l}\text { Vertical Intensity } \\
\text { for Alaska }\end{array}$} & \multicolumn{2}{|c|}{$\begin{array}{c}\text { Secular change in } \\
\text { declination for } \\
\text { Alaska }\end{array}$} & \multicolumn{2}{|c|}{$\begin{array}{l}\text { Secular change in } \\
\text { horizontal inten- } \\
\text { sity for Alaska }\end{array}$} & \multicolumn{2}{|c|}{$\begin{array}{r}\text { Secular change in } \\
\text { vertical inten- } \\
\text { s1ty for Alaska }\end{array}$} \\
\hline $\begin{array}{r}2.15098 \mathrm{e}+001 \\
-3.12571 \mathrm{e}-001 \\
4.91087 \mathrm{e}-001 \\
3.23674 \mathrm{e}-003 \\
-1.86390 \mathrm{e}-002\end{array}$ & $\begin{array}{l}1 \\
2 \\
3 \\
4 \\
5\end{array}$ & $\begin{array}{r}1.28701 \mathrm{e}+004 \\
5.18878 \mathrm{e}+002 \\
-1.00820 \mathrm{e}+002 \\
-9.02027 \mathrm{e}+000 \\
2.58613 \mathrm{e}+000\end{array}$ & $\begin{array}{l}1 \\
2 \\
3 \\
4 \\
5\end{array}$ & $\begin{array}{r}2.06571 \mathrm{e}+004 \\
-6.05428 \mathrm{e}+002 \\
1.97897 \mathrm{e}+002 \\
-9.99413 \mathrm{e}+000 \\
8.84446 \mathrm{e}+000\end{array}$ & $\begin{array}{l}1 \\
2 \\
3 \\
4 \\
5\end{array}$ & $\begin{array}{r}1.95174 \mathrm{e}-001 \\
-5.30394 \mathrm{e}-002 \\
-6.93826 \mathrm{e}-002 \\
-9.20386 \mathrm{e}-004 \\
-1.45384 \mathrm{e}-003\end{array}$ & $\begin{array}{l}1 \\
2 \\
3 \\
4 \\
5\end{array}$ & $\begin{array}{r}1.21089 \mathrm{e}+001 \\
-1.03493 \mathrm{e}+000 \\
2.69529 \mathrm{e}-001 \\
1.09158 \mathrm{e}-003 \\
7.47848 \mathrm{e}-003\end{array}$ & $\begin{array}{l}1 \\
2 \\
3 \\
4 \\
5\end{array}$ & $\begin{array}{r}-8.40721 e+000 \\
-8.68283 e-001 \\
3.36583 e-001 \\
1.22695 e-002 \\
-3.19064 e-002\end{array}$ & $\begin{array}{l}1 \\
2 \\
3 \\
4 \\
5\end{array}$ \\
\hline $\begin{array}{r}-5.69661 e-003 \\
2.77264 e-005 \\
4.11507 e-004 \\
-3.34078 e-004 \\
-1.72609 e-005\end{array}$ & $\begin{array}{r}6 \\
7 \\
8 \\
9 \\
10\end{array}$ & $\begin{array}{r}-1.40507 e+000 \\
-6.59361 \mathrm{e}-002 \\
2.72348 \mathrm{e}-001 \\
6.11470 \mathrm{e}-002 \\
-9.01411 \mathrm{e}-003\end{array}$ & $\begin{array}{r}6 \\
7 \\
8 \\
9 \\
10\end{array}$ & $\begin{array}{r}2.89759 e+000 \\
-2.26663 e-002 \\
-2.16847 e-001 \\
3.10657 e-002 \\
-3.77748 e-002\end{array}$ & $\begin{array}{r}6 \\
7 \\
8 \\
9 \\
10\end{array}$ & $\begin{array}{r}-1.03906 e-003 \\
-1.32668 e-006 \\
1.00093 e-004 \\
-4.85534 e-005 \\
-7.96214 e-007\end{array}$ & $\begin{array}{r}6 \\
7 \\
8 \\
9 \\
10\end{array}$ & $\begin{array}{r}9.37768 \mathrm{e}-003 \\
1.12332 \mathrm{e}-004 \\
-1.53755 \mathrm{e}-003 \\
2.18876 \mathrm{e}-004 \\
3.61790 \mathrm{e}-005\end{array}$ & $\begin{array}{r}6 \\
7 \\
8 \\
9 \\
10\end{array}$ & $\begin{array}{r}-4.80588 e-003 \\
1.02101 e-003 \\
-9.26915 e-004 \\
-8.09038 e-004 \\
8.37542 e-006\end{array}$ & $\begin{array}{r}6 \\
7 \\
8 \\
9 \\
10\end{array}$ \\
\hline $\begin{array}{r}1.38057 e-005 \\
-4.08427 e-006 \\
1.43227 e-005 \\
-3.78117 e-008 \\
3.43098 e-007\end{array}$ & $\begin{array}{r}11 \\
12 \\
13 \\
14 \\
15\end{array}$ & $\begin{array}{r}2.41091 e-003 \\
-6.30494 e-003 \\
4.71303 e-003 \\
-4.12616 e-004 \\
-1.14520 e-004\end{array}$ & $\begin{array}{l}11 \\
12 \\
13 \\
14 \\
15\end{array}$ & $\begin{array}{l}-2.56118 e-003 \\
-9.13153 e-003 \\
-6.19806 e-003 \\
-6.47016 e-004 \\
-6.21374 e-004\end{array}$ & $\begin{array}{l}11 \\
12 \\
13 \\
14 \\
15\end{array}$ & $\begin{array}{l}2.88554 \mathrm{e}-005 \\
4.68273 \mathrm{e}-006 \\
9.25196 \mathrm{e}-007 \\
2.22493 \mathrm{e}-007 \\
1.56201 \mathrm{e}-007\end{array}$ & $\begin{array}{l}11 \\
12 \\
13 \\
14 \\
15\end{array}$ & $\begin{array}{r}-1.25999 e-005 \\
-6.11094 e-005 \\
-2.82389 e-005 \\
9.39469 e-006 \\
-3.91379 e-006\end{array}$ & $\begin{array}{l}11 \\
12 \\
13 \\
14 \\
15\end{array}$ & $\begin{array}{r}1.56455 e-004 \\
1.11795 e-004 \\
-4.74751 \mathrm{e}-006 \\
-9.60527 \mathrm{e}-006 \\
3.87091 \mathrm{e}-006\end{array}$ & $\begin{array}{l}11 \\
12 \\
13 \\
14 \\
15\end{array}$ \\
\hline $\begin{array}{r}-1.45688 \mathrm{e}-006 \\
-1.41208 \mathrm{e}-006 \\
8.36353 \mathrm{e}-007 \\
-2.13940 \mathrm{e}-007 \\
1.92664 \mathrm{e}-007\end{array}$ & $\begin{array}{l}16 \\
17 \\
18 \\
19 \\
20\end{array}$ & $\begin{array}{r}-1.57527 e-005 \\
-3.36178 \mathrm{e}-004 \\
-9.66833 \mathrm{e}-005 \\
-7.09362 \mathrm{e}-005 \\
3.59370 \mathrm{e}-006\end{array}$ & $\begin{array}{l}16 \\
17 \\
18 \\
19 \\
20\end{array}$ & $\begin{array}{l}1.27664 e-003 \\
1.22739 e-004 \\
1.94955 e-004 \\
5.85795 e-005 \\
3.88750 e-006\end{array}$ & $\begin{array}{l}16 \\
17 \\
18 \\
19 \\
20\end{array}$ & $\begin{array}{r}1.06060 \mathrm{e}-007 \\
-1.23968 \mathrm{e}-007 \\
1.27469 \mathrm{e}-007 \\
-1.50581 \mathrm{e}-008 \\
-1.11625 \mathrm{e}-008\end{array}$ & $\begin{array}{l}16 \\
17 \\
18 \\
19 \\
20\end{array}$ & $\begin{array}{r}1.63643 e-006 \\
1.49433 e-006 \\
-3.68627 \mathrm{e}-007 \\
1.38704 \mathrm{e}-007 \\
-7.57077 \mathrm{e}-009\end{array}$ & $\begin{array}{l}16 \\
17 \\
18 \\
19 \\
20\end{array}$ & $\begin{array}{r}-1.91582 \mathrm{e}-006 \\
1.67819 \mathrm{e}-006 \\
4.45756 \mathrm{e}-007 \\
-4.37977 \mathrm{e}-007 \\
1.84588 \mathrm{e}-007\end{array}$ & $\begin{array}{l}16 \\
17 \\
18 \\
19 \\
20\end{array}$ \\
\hline $\begin{array}{r}-4.30511 \mathrm{e}-008 \\
-4.39980 \mathrm{e}-008 \\
1.99973 e-008 \\
-4.57007 \mathrm{e}-009 \\
2.50965 \mathrm{e}-009\end{array}$ & $\begin{array}{l}21 \\
22 \\
23 \\
24 \\
25\end{array}$ & $\begin{array}{r}3.09613 e-006 \\
7.94337 e-006 \\
9.40849 e-006 \\
-4.09482 e-006 \\
-1.35836 e-007\end{array}$ & $\begin{array}{l}21 \\
22 \\
23 \\
24 \\
25\end{array}$ & $\begin{array}{r}-3.02166 e-006 \\
8.37796 e-006 \\
3.91646 e-006 \\
5.44917 e-006 \\
1.43020 e-006\end{array}$ & $\begin{array}{l}21 \\
22 \\
23 \\
24 \\
25\end{array}$ & $\begin{array}{r}6.11890 e-009 \\
-6.18670 e-008 \\
1.76829 e-009 \\
-4.22369 e-009\end{array}$ & $\begin{array}{l}21 \\
22 \\
23 \\
24\end{array}$ & $\begin{array}{r}-7.56994 e-009 \\
-8.26998 e-009 \\
9.34528 \mathrm{e}-008 \\
3.68985 e-008\end{array}$ & $\begin{array}{l}21 \\
22 \\
23 \\
24\end{array}$ & $\begin{array}{r}2.30814 e-008 \\
-3.34610 e-007 \\
-9.34949 e-008 \\
4.87487 e-009\end{array}$ & $\begin{array}{l}21 \\
22 \\
23 \\
24\end{array}$ \\
\hline $\begin{array}{r}-2.70142 \mathrm{e}-009 \\
1.40864 \mathrm{e}-009 \\
-2.22711 \mathrm{e}-010 \\
9.37746 \mathrm{e}-010 \\
1.20184 \mathrm{e}-009\end{array}$ & $\begin{array}{l}26 \\
27 \\
28 \\
29 \\
30\end{array}$ & $\begin{array}{r}-1.35836 e-007 \\
-5.05727 e-007 \\
-2.22449 \mathrm{e}-007 \\
1.02968 \mathrm{e}-007 \\
-2.69126 \mathrm{e}-007 \\
2.12069 \mathrm{e}-007\end{array}$ & $\begin{array}{l}26 \\
27 \\
28 \\
29 \\
30\end{array}$ & $\begin{array}{r}1.43020 \mathrm{e}-006 \\
1.30237 \mathrm{e}-006 \\
-2.34036 \mathrm{e}-007 \\
1.68652 \mathrm{e}-007 \\
-2.24851 \mathrm{e}-006 \\
1.73023 \mathrm{e}-008\end{array}$ & $\begin{array}{l}26 \\
27 \\
28 \\
29 \\
30\end{array}$ & $\begin{array}{r}-9.96162 \mathrm{e}-011 \\
1.58122 \mathrm{e}-013 \\
-3.36216 \mathrm{e}-010 \\
7.60982 \mathrm{e}-011\end{array}$ & $\begin{array}{l}25 \\
26 \\
27 \\
28\end{array}$ & $\begin{array}{r}-8.15906 \mathrm{e}-009 \\
3.22362 \mathrm{e}-009 \\
-1.07443 \mathrm{e}-009 \\
7.79046 \mathrm{e}-010\end{array}$ & $\begin{array}{l}25 \\
26 \\
27 \\
28\end{array}$ & $\begin{array}{r}-1.72291 \mathrm{e}-008 \\
-4.20822 \mathrm{e}-009 \\
4.67631 \mathrm{e}-009 \\
-4.13954 \mathrm{e}-010\end{array}$ & $\begin{array}{l}25 \\
26 \\
27 \\
28\end{array}$ \\
\hline $\begin{array}{r}-1.83249 \mathrm{e}-010 \\
1.03445 \mathrm{e}-009 \\
-7.40522 \mathrm{e}-010 \\
9.24793 \mathrm{e}-011 \\
-2.09843 \mathrm{e}-011 \\
6.52480 \mathrm{e}-012\end{array}$ & $\begin{array}{l}31 \\
32 \\
33 \\
34 \\
35 \\
36\end{array}$ & $\begin{array}{r}1.68482 e-007 \\
5.70517 e-008 \\
-3.31915 e-009 \\
3.00740 e-008 \\
-5.55377 e-009 \\
-7.13698 e-010\end{array}$ & $\begin{array}{l}31 \\
32 \\
33 \\
34 \\
35 \\
36\end{array}$ & $\begin{array}{r}-2.78735 e-007 \\
-2.49108 e-008 \\
-8.86630 e-008 \\
-3.19313 e-009 \\
6.01991 e-009 \\
-3.49593 e-010\end{array}$ & $\begin{array}{l}31 \\
32 \\
33 \\
34 \\
35 \\
36\end{array}$ & & & & & & \\
\hline
\end{tabular}


